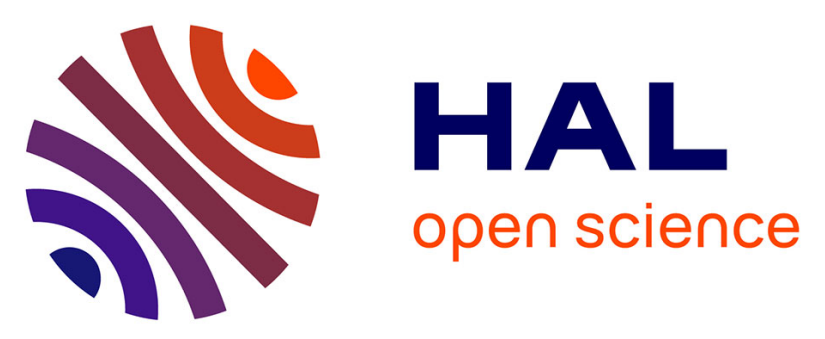

\title{
One-Pot, Solvent-Free Access to Unsymmetrical Ureas by Palladium-Catalysed Reductive Alkylation Using Molecular Hydrogen
}

\author{
Tharwat Mohy El Dine, Simon Chapron, Marie-Christine Duclos, Nicolas
}

Duguet, Florence Popowycz, Marc Lemaire

\section{To cite this version:}

Tharwat Mohy El Dine, Simon Chapron, Marie-Christine Duclos, Nicolas Duguet, Florence Popowycz, et al.. One-Pot, Solvent-Free Access to Unsymmetrical Ureas by Palladium-Catalysed Reductive Alkylation Using Molecular Hydrogen. European Journal of Organic Chemistry, 2013, 2013 (24), pp.5445-5454. 10.1002/ejoc.201300642 . hal-03249259

\section{HAL Id: hal-03249259 \\ https://univ-lyon1.hal.science/hal-03249259}

Submitted on 4 Jun 2021

HAL is a multi-disciplinary open access archive for the deposit and dissemination of scientific research documents, whether they are published or not. The documents may come from teaching and research institutions in France or abroad, or from public or private research centers.
L'archive ouverte pluridisciplinaire HAL, est destinée au dépôt et à la diffusion de documents scientifiques de niveau recherche, publiés ou non, émanant des établissements d'enseignement et de recherche français ou étrangers, des laboratoires publics ou privés. 


\title{
One-pot, solvent-free access to unsymmetrical ureas via palladium-catalyzed reductive alkylation using molecular hydrogen
}

\author{
Tharwat Mohy El Dine, ${ }^{[a]}$ Simon Chapron, ${ }^{[a]}$ Marie-Christine Duclos, ${ }^{[a]}$ Nicolas Duguet, ${ }^{[a]}$ \\ Florence Popowycz ${ }^{[\mathrm{b}]}$ and Marc Lemaire* ${ }^{*[a]}$
}

Keywords: Unsymmetrical disubstituted ureas / reductive alkylation / palladium / molecular hydrogen

Palladium-catalyzed reductive alkylation of monosubstituted ureas has been studied in the presence of aldehydes and molecular hydrogen as a clean reductant, giving unsymmetrical $N, N^{\prime}-$ disubstituted ureas with good to excellent isolated yields (60-93\%)

without the production of saline waste. [a] Equipe Catalyse, Synthèse et Environnement, Institut de Chimie et Biochimie Moléculaires et Supramoléculaires, UMR CNRS 5246, Université Claude Bernard-Lyon 1, Bâtiment Curien, 43 boulevard du 11 novembre 1918, 69622 Villeurbanne Cedex, France. Fax: +33(0)-472-43-14-08

Tel: +33(0)-472-43-14-07

E-mail: marc.lemaire.chimie@univ-lyon1.fr

[b] Equipe Chimie Organique et Bioorganique, Institut de Chimie et Biochimie Moléculaires et Supramoléculaires, UMR-CNRS 5246, Institut National des Sciences Appliquées (INSA Lyon), Bâtiment Jules Verne, 20 avenue Albert Einstein, 69621 Villeurbanne Cedex, France.

Fax: +33(0)-472-43-88-96

Tel: +33(0)-472-43-82-21

E-mail: florence.popowycz@insa-lyon.fr

Supporting information for this article is available on the WWW under http://dx.doi.org/10.1002/ejoc.xxxxxxxxx.

\section{Introduction}

Substituted ureas are encountered in numerous biologically active compounds such as pharmaceuticals (i.e. Sorafenib, Cariprazine) and agrochemicals (i.e. Monuron, Isoproturon, Diuron). ${ }^{[1]}$ For example, they have recently been identified as potential anti-nociceptive, ${ }^{[2]}$ anti-glycating ${ }^{[3]}$ and anti-cancer ${ }^{[4]}$ agents. Urea derivatives are also used as substrates in material science, intermediates in macromolecular synthesis, linkers in combinatorial chemistry and as organocatalysts. ${ }^{[5]}$ Recently, urea chemistry has gained a renewal of interest thanks to the unearthing of new reactivity and the development of modern synthetic methods. ${ }^{[6-9]}$

Ureas are traditionally prepared from phosgene or commercially available isocyanates usually providing symmetrical and unsymmetrical products, respectively. Alternatively, isocyanates could be also generated in situ through Curtius, Lossen or Hoffmann rearrangements. Despite the fact that the toxicity and the dangerousness of these reagents have been demonstrated for decades, these methods are still widely used in industry. Intense efforts have been directed towards the replacement of these hazardous reagents by safer and environmentally-friendly substitutes such as triphosgene, ${ }^{[10]}$ carbonyldiimidazole ${ }^{[11]}$ and related compounds, ${ }^{[12]}$ chloroformates, ${ }^{[13]}$ carbamates, ${ }^{[14]}$
This reaction was incorporated to a one-pot, solvent-free sequence involving the alkylation of in-situ generated monosubstituted ureas from the corresponding amines. carbonates, ${ }^{[15]}$ among others. ${ }^{[16]}$ Transition-metal catalyzed carbonylation of amines with $\mathrm{CO}$ has been reported as an excellent atom-economy pathway but the toxicity of this gas compromises the development of this route. ${ }^{[17]}$ Finally, the most promising alternative is probably the use of carbon dioxide as a carbonylating agent. However, up to now this methodology is limited to the preparation of symmetrical ureas. ${ }^{[18]}$

The access to unsymmetrical ureas can be achieved from the functionalization of monosubstituted ureas via $\mathrm{N}$-arylation or $\mathrm{N}$ alkylation. If several efficient methods of (hetero)arylation have already been reported, ${ }^{[19]}$ this is not the case for alkylation that has been scarcely studied. Early methods relying on the use of alkyl halides or pseudohalides gave low yields due to the competing $O$ alkylation. $N$-Alkylation could be attained under phase-transfer catalysis but only alkyl ureas could be used as starting materials. ${ }^{[20]}$ Finally, the selective $N$-alkylation could be achieved by reductive alkylation of monosubstituted ureas using aldehydes as alkylating agents and hydrides as hydrogen source. Only three methods have been reported so far, unfortunately, none of them allows the use of enolizable aldehydes. ${ }^{[21-22]}$ Moreover, it is often necessary to form the imine prior to the reduction step and to use the urea in excess in order to avoid over alkylation. The major drawback of these methodologies lies in the use of a large excess of hydrides leading to the production of large quantities of waste and rendering the work-up tedious.

For several years, our laboratory has been interested in developing green and sustainable reductive alkylation methods using molecular hydrogen as reducing agent. Thus, amides, ${ }^{[23]}$ alcohols ${ }^{[24]}$ and polyols, ${ }^{[25]}$ such as glycerol, ${ }^{[26]}$ have been alkylated without the production of salts. Numerous methods using hydrogen have already been reported in the field of reductive amination ${ }^{[27]}$ but, to the best of our knowledge, $N$-alkylation of ureas has never been described under such conditions. We report here our efforts to develop the selective reductive alkylation of monosubstituted ureas with aldehydes and molecular hydrogen as clean reductant. The inclusion of this procedure in a one-pot, solvent-free sequence to prepare unsymmetrical $N, N^{\prime}$-disubstituted ureas from the corresponding amines is also described.

\section{Results and Discussion}




\section{Preparation of monosubstituted ureas}

We have recently showed that trimethylsilylisocyanate (TMSNCO) is the main by-product of HMDS-catalyzed trimerization of alkylisocyanates. ${ }^{[28]}$ As this route is the privileged one for the production of isocyanurates, tons of this compound are generated each year. Unfortunately, no economically-viable valorization process has been identified to date. It should be noted that trimethylsilylisocyanate can be viewed as an equivalent of isocyanic acid but with a lower volatility and an attenuated toxicity Thus, the recycling of trimethylsilylisocyanate for the preparation of monosubstituted ureas was a determining step in this project. The addition of TMS-NCO to a range of commercially available (both aliphatic and aromatic) primary amines 1-7 gave the corresponding silylated ureas under solvent-free conditions. These intermediates were not isolated but submitted to methanolysis furnishing the corresponding ureas 8-14 with good to excellent isolated yields (70-93\%) (Figure 1).

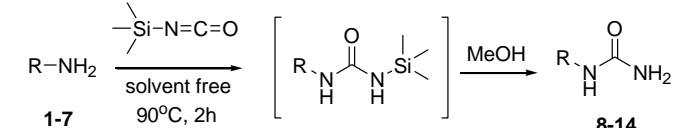

$$
\begin{aligned}
& \underset{8(93 \%)}{(87 \%)} \\
& { }_{12(85 \%)}
\end{aligned}
$$

Figure 1. Preparation of a range of monosubstituted ureas.

\section{Reductive alkylation of monosubstituted ureas}

Initial studies focused upon the demonstration that monosubstituted ureas could be alkylated using aldehydes and molecular hydrogen as reducing agent. Thus, treatment of benzylurea 12 with decanal 15 (5 equiv) under reductive alkylation conditions (5\%-Pd/C (10 mol-\%), $\mathrm{H}_{2}$ (20 bar), $\mathrm{Na}_{2} \mathrm{SO}_{4}$ (2 equiv) in anhydrous $\mathrm{MeOH}$ at $100{ }^{\circ} \mathrm{C}$ ) gave the corresponding urea 16 with a satisfying $60 \%$ yield. In this protocol, sodium sulfate was initially chosen for its dehydrating properties but could also act as a catalyst poison to prevent the reduction of the aldehyde. Unfortunately, the contaminant formation of decanol could not be avoided and the relatively harsh conditions also led to the formation of other byproducts (such as debenzylation and aromatic ring reduction) (Scheme 1).
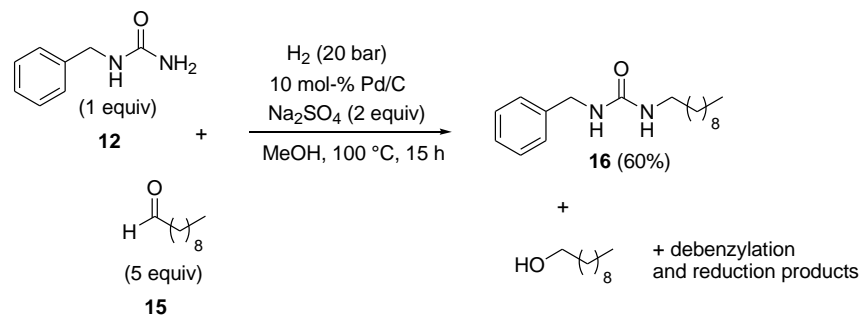

Scheme 1. Preliminary result for reductive alkylation of benzylurea.

Optimization studies were carried out in order to find milder conditions aiming to make this protocol suitable for both aliphatic and aromatic aldehydes. For that purpose, aliphatic hexylurea 8 was chosen as a model substrate and the reactions were typically carried out using decanal $\mathbf{1 5}$ in the presence of $\mathrm{H}_{2}, 5 \%-\mathrm{Pd} / \mathrm{C}$ and $\mathrm{Na}_{2} \mathrm{SO}_{4}$ (2 equiv.) in dry methanol at $100{ }^{\circ} \mathrm{C}$ for $15 \mathrm{~h}$ (Table 1 ).

Table 1. Optimization of reaction conditions. ${ }^{[\mathrm{a}]}$

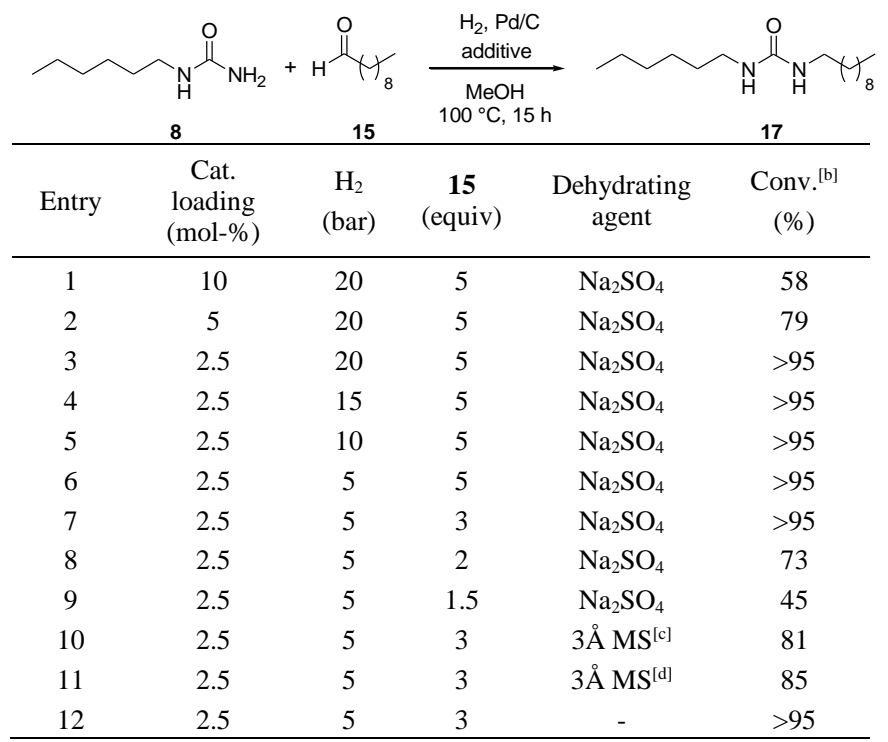

[a] Reaction conditions: $1 \mathrm{mmol}$ of $\mathbf{8}, \mathbf{1 5}, 5 \%-\mathrm{Pd} / \mathrm{C}, \mathrm{H}_{2}$, dehydrating agent (2 equiv.), $10 \mathrm{~mL} \mathrm{MeOH}, 100{ }^{\circ} \mathrm{C}, 15 \mathrm{~h}$. [b] Determined by ${ }^{1} \mathrm{H}$ NMR. [c] $200 \mathrm{mg}$ (pellets). [d] $200 \mathrm{mg}$ (grinded).

The influence of the catalyst loading was first probed. High catalyst loadings resulted in partial conversion of the starting material (Table 1, Entries 1-2). This result was attributed to the fact that hydrogenation of the aldehyde was favoured under these conditions, thus limiting the amount available for the formation of the urea-aldehyde adduct. Decreasing the loading to 2.5 mol- $\%$ permitted to reach quantitative conversion of hexylurea to the corresponding alkylated urea 17 (Table 1, Entry 3). Keeping in mind that aromatic aldehydes will be also used as alkylating agents, the hydrogen pressure was progressively decreased from 20 to 5 bar. No significant impact on the conversion was observed (Table 1, Entries 3-6). No attempt was made to decrease the hydrogen pressure down to 1 bar for technical and safety reasons. ${ }^{[29]}$ Reducing the aldehyde amount to 3 equivalents did not affect the conversion (Table 1, Entry 7). Unfortunately, further decrease to 2 and 1.5 equivalents did not permit to reach the total consumption of the starting material with 73 and $45 \%$ conversion, respectively (Table 1, Entries 8-9). Since the utilization of $\mathrm{Na}_{2} \mathrm{SO}_{4}$ as a dehydrating agent and/or catalyst poison leads to the production of waste, we attempted to replace it by $3 \AA$ molecular sieves. Conversions of about $80 \%$ were observed in the presence of molecular sieves, whether as pellets or as grinded (Table 1, Entries 10-11). Finally, a control experiment revealed that a dehydrating agent was not necessary to reach high conversion under these conditions (Table 1, Entry 12). This intriguing result shows that the presence of water is not detrimental for the reaction process. This could be explained by the potential dehydrating effect of dry methanol that allows the formation of an imine from the corresponding hemi-aminal. Subsequent hydrogenation would lead to the desired $N, N^{\prime}$-disubstituted urea. However, an alternative mechanism pathway involving the hydrogenolysis of the hemiaminal could not be ruled out under these conditions (Scheme 2). ${ }^{[27 a]}$ 


$$
\text { Hydrogenolysis }
$$

Scheme 2. Potential mechanism pathways.

Treatment of hexylurea $\mathbf{8}$ with 3 equivalents of decanal 15 under optimized conditions (5 bar $\mathrm{H}_{2}, 2.5 \mathrm{~mol}-\% \mathrm{Pd} / \mathrm{C}, 100{ }^{\circ} \mathrm{C}$ in $\mathrm{MeOH}$ ) gave the corresponding decylhexylurea 17 with $88 \%$ isolated yield (Scheme 3).

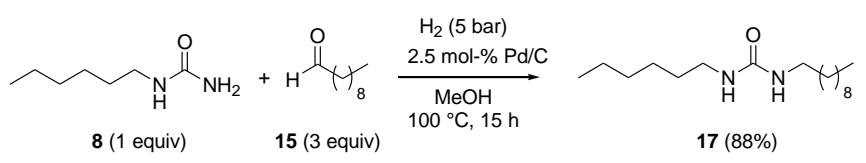

Scheme 3. Reductive alkylation under optimized conditions.

The generality of the reductive alkylation of monosubstituted ureas was next established using hexylurea $\mathbf{8}$ as model substrate and a range of aliphatic and aromatic aldehydes, furnishing the corresponding $N, N^{\prime}$-disubstituted ureas $\mathbf{1 7}, \mathbf{2 5}-\mathbf{3 1}$ in $65-93 \%$ isolated yields (Table 2). A slight drop of yield was observed when decreasing the chain length of aliphatic aldehydes from 12 to 4 carbons (Table 2, Entries 1-6). Propionaldehyde and acetaldehyde could also be used as alkylating agents, however, isolation and purification of the corresponding ureas proved to be laborious. Methylation with aqueous formaldehyde or paraformaldehyde failed to give hexylmethylurea and led to the formation of complex mixtures. Aryl- and alkylaryl- aldehydes were also tolerated, giving ureas $\mathbf{3 0}$ and $\mathbf{3 1}$ in 93 and $90 \%$ isolated yields, respectively (Table 2, Entries 7-8). It should be noted that neither debenzylation nor aromatic ring reduction were observed under these conditions.

Table 2. Scope of aldehydes. ${ }^{\text {[a] }}$

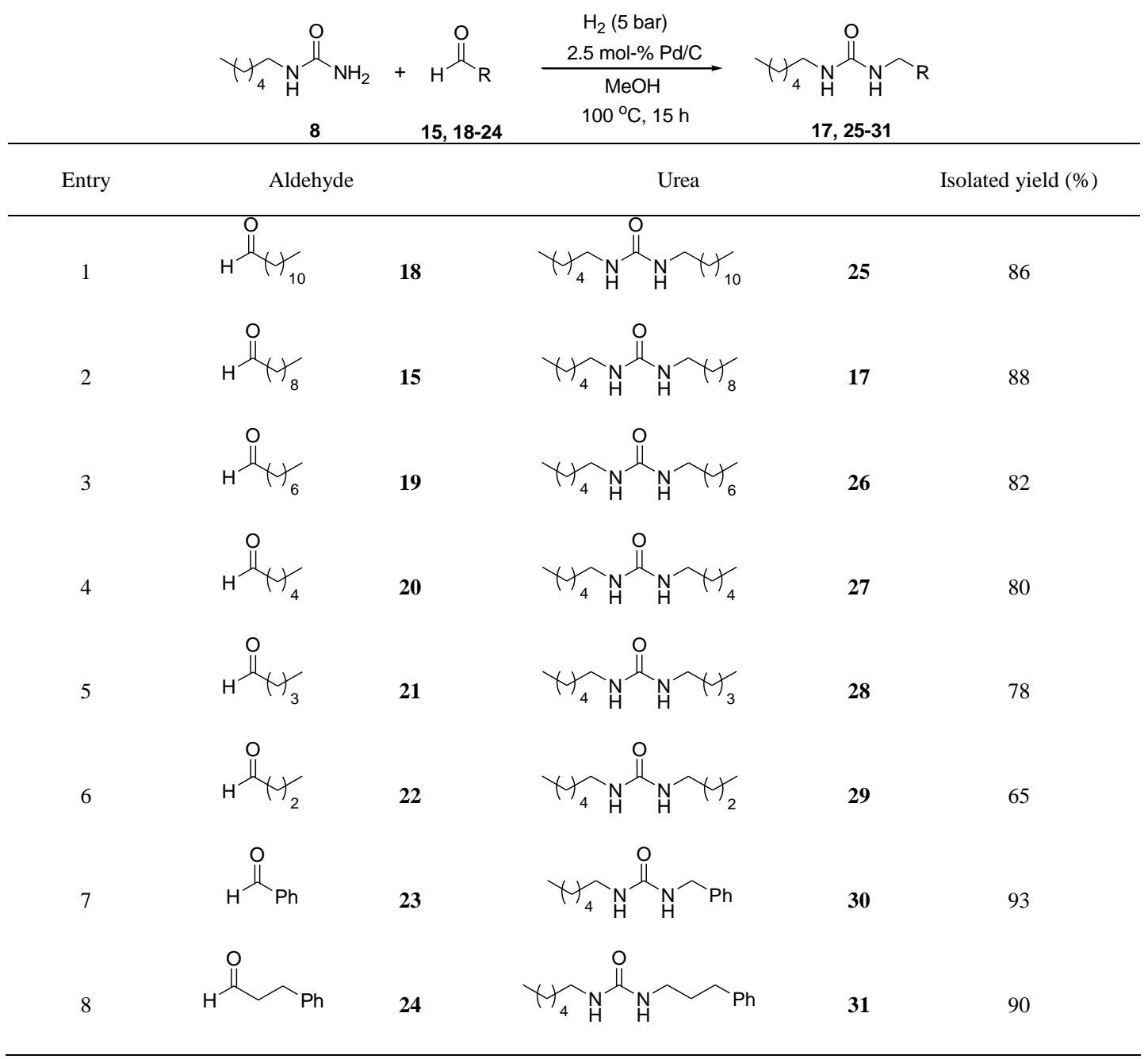

[a] Reaction conditions: $1 \mathrm{mmol}$ of $\mathbf{8}, 3 \mathrm{mmol}$ of aldehyde, $\mathrm{H}_{2}(5 \mathrm{bar}), 5 \%-\mathrm{Pd} / \mathrm{C}(2.5 \mathrm{~mol}-\%), \mathrm{MeOH}(10 \mathrm{~mL}), 100{ }^{\circ} \mathrm{C}, 15 \mathrm{~h}$.

The substrate scope was next examined under optimized conditions (5 bar of $\mathrm{H}_{2}, 2.5 \mathrm{~mol}-\%$ of $5 \%-\mathrm{Pd} / \mathrm{C}, 100{ }^{\circ} \mathrm{C}$ in $\mathrm{MeOH}$ ) using decanal as alkylating agent and a range of monosubstituted aliphatic, benzylic and aromatic ureas. The corresponding 
unsymmetrical $N, N^{\prime}$-disubstituted ureas $\mathbf{1 6}, \mathbf{3 3}-\mathbf{3 8}$ were isolated in 60-86\% yields (Table 3 ). Commercially available methylurea 32 was alkylated with only $60 \%$ yield (Table 3, Entry 1) but other alkyl-, cycloalkyl- and alkylaryl-ureas were efficiently converted to their corresponding disubstituted ureas with good yields (78-80\%) (Table 3, Entries 2-4). Benzylic ureas 12 and $\mathbf{1 3}$ were also subjected to reductive alkylation conditions without debenzylation, affording ureas $\mathbf{1 6}$ and $\mathbf{3 7}$ with 86 and $72 \%$ yield, respectively (Table 3, Entries 5-6). Finally, aromatic urea 14 was also tolerated, generating the corresponding alkylarylurea $\mathbf{3 8}$ with $65 \%$ yield (Table 3, Entry 7).

Table 3. Scope of ureas. ${ }^{[a]}$

\begin{tabular}{|c|c|c|c|c|c|c|}
\hline & $9-14,32$ & & $\begin{array}{c}\begin{array}{c}\mathrm{H}_{2}(5 \mathrm{bar}) \\
2.5 \mathrm{~mol}-\% \mathrm{Pd} / \mathrm{C}\end{array} \\
\underset{\mathrm{MeOH}}{100^{\circ} \mathrm{C}, 15 \mathrm{~h}}\end{array}$ & ${ }_{16,33-38}$ & & \\
\hline Entry & & & & Alkylated urea & & $\begin{array}{c}\text { Isolated } \\
\text { yield (\%) }\end{array}$ \\
\hline 1 & & 32 & & & 33 & 86 \\
\hline 2 & & 9 & & & 34 & 88 \\
\hline 3 & & 10 & & & 35 & 82 \\
\hline 4 & & 11 & & & 36 & 80 \\
\hline 5 & & 12 & & & 16 & 78 \\
\hline 6 & $\mathrm{H}$ & 13 & & & 37 & 65 \\
\hline 7 & & 14 & & & 38 & 90 \\
\hline
\end{tabular}

[a] Reaction conditions: $1 \mathrm{mmol}$ of urea, $3 \mathrm{mmol}$ of decanal 15, $\mathrm{H}_{2}(5 \mathrm{bar}), 5 \%-\mathrm{Pd} / \mathrm{C}(2.5 \mathrm{~mol}-\%), \mathrm{MeOH}(10 \mathrm{~mL}), 100{ }^{\circ} \mathrm{C}, 15 \mathrm{~h}$

\section{One-pot, solvent-free access to unsymmetrical ureas}

As monosubstituted ureas were prepared through the addition of trimethylsilylisocyanate to primary amines followed by methanolysis, we envisioned that the reductive alkylation could be directly realized on silylated ureas, without the need of isolating these intermediates. Furthermore, it was also envisioned that the whole procedure could be carried out under solvent-free conditions. To that purpose, the optimized reductive alkylation method was first checked under solvent-free conditions. Treatment of hexylurea with decanal gave the corresponding urea $\mathbf{1 7}$ with $80 \%$ isolated yield, confirming the robustness of this protocol (Scheme 4).



Scheme 4. Reductive alkylation under solvent-free conditions.
The one-pot, solvent-free procedure was next probed (Table 4) Treatment of hexylamine $\mathbf{1}$ with trimethylsilylisocyanate at $90{ }^{\circ} \mathrm{C}$ for $2 \mathrm{~h}$ gave the corresponding silylated urea. This intermediate was directly treated with decanal $\mathbf{1 5}$ in the presence of $2.5 \mathrm{~mol}-\%$ $\mathrm{Pd} / \mathrm{C}$ and 5 bar of $\mathrm{H}_{2}$ at $100{ }^{\circ} \mathrm{C}$ for $15 \mathrm{~h}$ furnishing decylhexylurea 17 with $60 \%$ isolated yield (Table 4, Entry 1). This one-pot procedure also allowed amines $\mathbf{2}-\mathbf{5}$ and 4-methoxy-aniline $\mathbf{7}$ to be readily converted to the corresponding unsymmetrical $N, N^{\prime}$ disubstituted ureas in 45-63\% isolated yields (Table 4, Entries 26). Notably, benzylamine could be turned into the corresponding benzyldecylurea $\mathbf{1 6}$ under these conditions without the formation of any debenzylation or aromatic ring reduction side-products (Table 4, Entry 5). 




[a] Reaction conditions: $10 \mathrm{mmol}$ of amine, $12 \mathrm{mmol}$ of TMS-NCO, $90{ }^{\circ} \mathrm{C}, 2 \mathrm{~h}$, then, $30 \mathrm{mmol}$ of decanal $\mathbf{1 5}, \mathrm{H}_{2}(5 \mathrm{bar}), 5 \%$ $\mathrm{Pd} / \mathrm{C}(2.5 \mathrm{~mol}-\%), 100{ }^{\circ} \mathrm{C}, 15 \mathrm{~h}$.

\section{Conclusions}

In summary, we described the palladium-catalyzed reductive alkylation of monosubstituted ureas using aldehydes as alkylating agents and molecular hydrogen as clean reductant. This method afforded the corresponding unsymmetrical $N, N^{\prime}$-disubstituted ureas with good to excellent isolated yields (60-93\%). In contrary to the previously reported methods using hydrides, such as $\mathrm{NaBH}_{4}$ and $\mathrm{Et}_{3} \mathrm{SiH}$, this system allows the selective $\mathrm{N}$-alkylation with both aliphatic and aromatic aldehydes. This protocol was then extended to the one-pot, solvent-free preparation of unsymmetrical $N, N^{\prime}$ disubstituted ureas from amines through reductive alkylation of the corresponding silylated ureas.

\section{Experimental Section}

\section{General information}

All reagents and solvents used for synthesis were commercial, used without further purification and supplied by Aldrich, Acros, Lancaster, Alfa Aesar and Fluka. NMR spectra were acquired on either Bruker $300\left({ }^{1} \mathrm{H}, 300\right.$ $\left.\mathrm{MHz} ;{ }^{13} \mathrm{C}, 75 \mathrm{MHz}\right)$ or Bruker $400\left({ }^{1} \mathrm{H}, 400 \mathrm{MHz},{ }^{13} \mathrm{C}, 100 \mathrm{MHz}\right)$ or Bruker $500\left({ }^{1} \mathrm{H}, 500 \mathrm{MHz},{ }^{13} \mathrm{C}, 125 \mathrm{MHz}\right)$ spectrometer at $293 \mathrm{~K}$. Shifts are referenced relative to the deuterated solvent residual peak. The chemical shifts $(\delta)$ are expressed in ppm and the coupling constants $(J)$ are given in
Hz. The following abbreviations are used to explain the multiplicities: $\mathrm{s}=$ singlet, $\mathrm{d}=$ doublet, $\mathrm{t}=$ triplet, $\mathrm{q}=$ quartet, $\mathrm{qt}=$ quintet, $\mathrm{m}=$ multiplet, $\mathrm{br}=$ broad. Electrospray ionization (ESI) mass spectra (MS) and HighResolution Mass Spectra (HRMS) were recorded in the positive mode using a Bruker MicrOTOF-Q II XL spectrometer. Thin-layer chromatography (TLC) was carried out on aluminum sheets coated with silica gel Merck 60 F254 $(0.25 \mathrm{~mm})$. Flash column chromatography was performed with silica gel Merck Si $60(40-63 \mu \mathrm{m})$. Infra-red (IR) spectra were recorded in a SMART iTR-Nicolet iS10 spectrometer using Attenuated Total Reflectance (ATR) and the wavenumbers ( $v$ max) are expressed in $\mathrm{cm}^{-1}$. Melting points were measured using a Banc Kofler apparatus and noted in ${ }^{\circ} \mathrm{C}$.

Procedure A: General procedure for the preparation of monosubstituted ureas.

Trimethylsilylisocyanate (1.2 equiv) was added to an aliphatic or aromatic primary amine (1.0 equiv, neat) in a sealed tube at room temperature and the mixture was stirred at $90{ }^{\circ} \mathrm{C}$ for 2 hours unless otherwise stated. The reaction mixture was cooled, treated with methanol and the solvent was removed under reduced pressure. The residue was purified by recrystallization from $\mathrm{EtOH} / \mathrm{Et}_{2} \mathrm{O}$

Hexylurea (8). The title compound was prepared from hexylamine (1.31 $\mathrm{mL}, 9.85 \mathrm{mmol})$ following the procedure $\mathbf{A}$ to give $\mathbf{8}(1.32 \mathrm{~g}, 93 \%$ yield) as a white crystalline solid. m.p.: $112{ }^{\circ} \mathrm{C} ;{ }^{1} \mathrm{H}$ NMR (300 MHz, $d_{6}$-DMSO) $\delta$ 
$0.85(3 \mathrm{H}, \mathrm{t}, J=6.7), 1.10-1.50(8 \mathrm{H}, \mathrm{m}), 2.93(2 \mathrm{H}, \mathrm{dt}$ app q, $J=6.5), 5.42$ $\left(2 \mathrm{H}, \mathrm{s}, \mathrm{NH}_{2}\right), 5.94(1 \mathrm{H}, \mathrm{t}, J=5.6, \mathrm{NH}) ;{ }^{13} \mathrm{C}$ NMR $\left(75 \mathrm{MHz}, d_{6}\right.$-DMSO) $\delta$ $13.9\left(\mathrm{CH}_{3}\right), 22.2\left(\mathrm{CH}_{2}\right), 26.2\left(\mathrm{CH}_{2}\right), 30.0\left(\mathrm{CH}_{2}\right), 31.2\left(\mathrm{CH}_{2}\right), 39.2\left(\mathrm{CH}_{2}\right)$, $159.0(\mathrm{Cq})$; IR (ATR) v max: $3391(\mathrm{~N}-\mathrm{H}), 3197(\mathrm{~N}-\mathrm{H}), 2949,2932,2867$, $1655(\mathrm{C}=\mathrm{O}), 1629,1600,1530,1480,1468,1452,1323,1150,780,728$; MS $\left(\mathrm{ESI}^{+}\right) \mathrm{m} / \mathrm{z} 145\left([\mathrm{M}+\mathrm{H}]^{+}, 51\right), 289\left([2 \mathrm{M}+\mathrm{H}]^{+}, 100\right)$.

Cyclohexylurea (9). The title compound was prepared from cyclohexylamine $(1.2 \mathrm{~mL}, 10.38 \mathrm{mmol})$ following the procedure $\mathbf{A}$ to give $9(1.29 \mathrm{~g}, 87 \%$ yield $)$ as a white crystalline solid. m.p.: $205{ }^{\circ} \mathrm{C} ;{ }^{1} \mathrm{H}$ NMR (300 MHz, $d_{6}$-DMSO) $\delta 0.95-1.38(5 \mathrm{H}, \mathrm{m}), 1.40-1.88(5 \mathrm{H}, \mathrm{m}), 3.20-3.40$ $(1 \mathrm{H}, \mathrm{m}), 5.28\left(2 \mathrm{H}, \mathrm{s}, \mathrm{NH}_{2}\right), 5.81(1 \mathrm{H}, \mathrm{d}, J=7.9, \mathrm{NH}) ;{ }^{13} \mathrm{C} \mathrm{NMR}(75 \mathrm{MHz}$, $d_{6}$-DMSO) $\delta 24.6\left(2 \mathrm{CH}_{2}\right), 25.4\left(\mathrm{CH}_{2}\right), 33.4\left(2 \mathrm{CH}_{2}\right), 47.7(\mathrm{CH}), 158.3$ $(\mathrm{Cq})$; IR (ATR) $\vee$ max: $3421(\mathrm{~N}-\mathrm{H}), 3327(\mathrm{~N}-\mathrm{H}), 3196(\mathrm{~N}-\mathrm{H}), 2928,2852$, 1649 (C=O), 1620, 1595, 1544, 1446, 1384, 1351, 1257, 1157, 892, 779; MS $\left(\mathrm{ESI}^{+}\right) \mathrm{m} / z 143\left([\mathrm{M}+\mathrm{H}]^{+}, 76\right), 285\left([2 \mathrm{M}+\mathrm{H}]^{+}, 100\right)$.

1-(Cyclohexylmethyl)urea (10). The title compound was prepared from 1(cyclohexylmethyl)amine $(1.3 \mathrm{~mL}, 9.79 \mathrm{mmol})$ following the procedure $\mathbf{A}$ to give $\mathbf{1 0}(1.33 \mathrm{~g}, 87 \%$ yield) as a white crystalline powder. m.p.: 180 $181{ }^{\circ} \mathrm{C}$; ${ }^{1} \mathrm{H}$ NMR $\left(300 \mathrm{MHz}, d_{6}\right.$-DMSO) $\delta 0.70-0.96(2 \mathrm{H}, \mathrm{m}), 0.98-1.41$ $(4 \mathrm{H}, \mathrm{m}), 1.50-1.78(5 \mathrm{H}, \mathrm{m}), 2.79(2 \mathrm{H}, \mathrm{dd}$ app t, $J=6.3), 5.32\left(2 \mathrm{H}, \mathrm{s}, \mathrm{NH}_{2}\right)$, $5.92(1 \mathrm{H}, \mathrm{t}, J=5.4, \mathrm{NH}) ;{ }^{13} \mathrm{C} \mathrm{NMR}\left(75 \mathrm{MHz}, d_{6}\right.$-DMSO) $\delta 25.5\left(2 \mathrm{CH}_{2}\right)$, $26.2\left(\mathrm{CH}_{2}\right), 30.4\left(2 \mathrm{CH}_{2}\right), 38.2(\mathrm{CH}), 45.5\left(\mathrm{CH}_{2}\right), 158.9(\mathrm{Cq})$; IR (ATR) v max: $3384(\mathrm{~N}-\mathrm{H}), 3198(\mathrm{~N}-\mathrm{H}), 2920,2851,1651(\mathrm{C}=\mathrm{O}), 1606,1546,1451$ $1433,1379,1155,959,782 ; \mathrm{MS}_{\left(\mathrm{ESI}^{+}\right)} \mathrm{m} / z 157\left([\mathrm{M}+\mathrm{H}]^{+}, 61\right), 313([2 \mathrm{M}+$ $\left.\mathrm{H}]^{+}, 100\right)$.

Phenylethylurea (11). The title compound was prepared from 2phenylethylamine $(1.25 \mathrm{~mL}, 9.84 \mathrm{mmol})$ following the procedure $\mathbf{A}$ to give $11(1.13 \mathrm{~g}, 70 \%$ yield $)$ as a white crystalline solid. m.p.: $112-114{ }^{\circ} \mathrm{C} ;{ }^{1} \mathrm{H}$ NMR (300 MHz, $d_{6}$-DMSO) $\delta 2.66$ ( $\left.2 \mathrm{H}, \mathrm{t}, J=7.2\right), 3.19$ (2H, dt app q, $J=$ 6.8), $5.42\left(2 \mathrm{H}, \mathrm{s}, \mathrm{NH}_{2}\right), 5.90(1 \mathrm{H}, \mathrm{t}, J=5.4, \mathrm{NH}), 7.15-7.25\left(3 \mathrm{H}^{\mathrm{ar}}, \mathrm{m}\right)$, 7.25-7.35 (2H $\left.{ }^{\text {ar }}, \mathrm{m}\right) ;{ }^{13} \mathrm{C}$ NMR $\left(75 \mathrm{MHz}, d_{6}\right.$-DMSO) $\delta 36.2\left(\mathrm{CH}_{2}\right), 40.9$ $\left(\mathrm{CH}_{2}\right), 126.0(\mathrm{CH}), 128.3(2 \mathrm{CH}), 128.7(2 \mathrm{CH}), 139.8(\mathrm{Cq}), 158.9(\mathrm{Cq}) ; \mathrm{IR}$ (ATR) v max: $3420(\mathrm{~N}-\mathrm{H}), 3335(\mathrm{~N}-\mathrm{H}), 3213(\mathrm{~N}-\mathrm{H}), 1650(\mathrm{C}=\mathrm{O}), 1598$,

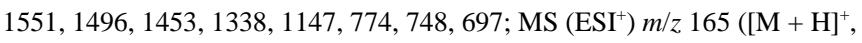
$100), 187\left([\mathrm{M}+\mathrm{Na}]^{+}, 21\right), 329\left([2 \mathrm{M}+\mathrm{H}]^{+}, 34\right), 351\left([2 \mathrm{M}+\mathrm{Na}]^{+}, 17\right)$.

Benzylurea (12). The title compound was prepared from benzylamine (1.1 $\mathrm{mL}, 10.1 \mathrm{mmol}$ ) following the procedure $\mathbf{A}$ to give $\mathbf{1 2}(1.37 \mathrm{~g}, 85 \%$ yield $)$ as a white crystalline solid. m.p.: $151{ }^{\circ} \mathrm{C} ;{ }^{1} \mathrm{H}$ NMR $\left(300 \mathrm{MHz}, d_{6}\right.$-DMSO) $\delta$ $4.25(2 \mathrm{H}, \mathrm{d}, J=5.9), 5.81\left(2 \mathrm{H}, \mathrm{s}, \mathrm{NH}_{2}\right), 6.63(1 \mathrm{H}, \mathrm{t}, J=5.9, \mathrm{NH}), 7.12-$ $7.47\left(5 \mathrm{H}^{\mathrm{ar}}, \mathrm{m}\right) ;{ }^{13} \mathrm{C}$ NMR $\left(75 \mathrm{MHz}, d_{6}\right.$-DMSO) $\delta 43.1\left(\mathrm{CH}_{2}\right), 126.8(\mathrm{CH})$, $127.2(2 \mathrm{CH}), 128.4(2 \mathrm{CH}), 141.0(\mathrm{Cq}), 159.4(\mathrm{Cq})$; IR (ATR) v max: $3426(\mathrm{~N}-\mathrm{H}), 3328(\mathrm{~N}-\mathrm{H}), 1647(\mathrm{C}=\mathrm{O}), 1597,1556,1467,1455,1386$, 1328, 1309, 1142, 1107, 750, 694; MS (ESI $\left.{ }^{+}\right) \mathrm{m} / z .151\left([\mathrm{M}+\mathrm{H}]^{+}, 100\right), 173$ $\left([\mathrm{M}+\mathrm{Na}]^{+}, 29\right), 301\left([2 \mathrm{M}+\mathrm{H}]^{+}, 48\right), 323\left([2 \mathrm{M}+\mathrm{Na}]^{+}, 24\right)$.

1-(1-Phenylethyl)urea (13). The title compound was prepared from 1phenylethylamine $(1.3 \mathrm{~mL}, 10.1 \mathrm{mmol})$ following the procedure $\mathbf{A}$ to give 13 (1.42 g, 85\% yield) as a white powder. m.p.: $117{ }^{\circ} \mathrm{C} ;{ }^{1} \mathrm{H}$ NMR $(300$ $\mathrm{MHz}, d_{6}$-DMSO) $\delta 1.34(3 \mathrm{H}, \mathrm{d}, J=6.6), 4.78(1 \mathrm{H}, \mathrm{t}, J=6.8), 5.62(2 \mathrm{H}, \mathrm{s}$, $\left.\mathrm{NH}_{2}\right), 6.56(1 \mathrm{H}, \mathrm{d}, J=7.7, \mathrm{NH}), 7.00-7.67\left(5 \mathrm{H}^{\mathrm{ar}}, \mathrm{m}\right) ;{ }^{13} \mathrm{C} \mathrm{NMR}(75 \mathrm{MHz}$, $d_{6}$-DMSO) $\delta 23.4\left(\mathrm{CH}_{3}\right), 48.7(\mathrm{CH}), 125.9(2 \mathrm{CH}), 126.5(\mathrm{CH}), 128.3(2$ $\mathrm{CH}), 145.9(\mathrm{Cq}), 158.4(\mathrm{Cq})$; IR (ATR) $v$ max: $3418(\mathrm{~N}-\mathrm{H}), 3328(\mathrm{~N}-\mathrm{H})$, 3208 (N-H), 2975, 1647 (C=O), 1593, 1533, 1494, 1450, 1372, 1279, 1148 1021, 900, 749, 695; $\mathrm{MS}\left(\mathrm{ESI}^{+}\right) \mathrm{m} / \mathrm{z} 187\left([\mathrm{M}+\mathrm{Na}]^{+}, 32\right), 351\left([2 \mathrm{M}+\mathrm{Na}]^{+}\right.$, $36)$.

4-Methoxy-phenylurea (14). The title compound was prepared from $p$ anisidine $(1.23 \mathrm{~g}, 10 \mathrm{mmol})$ following the procedure A to give $\mathbf{1 4}(1.50 \mathrm{~g}$, $90 \%$ yield) as a white crystalline solid. m.p.: $78-79{ }^{\circ} \mathrm{C} ;{ }^{1} \mathrm{H}$ NMR $(300 \mathrm{MHz}$,
$d_{6}$-DMSO) $\delta 3.68(3 \mathrm{H}, \mathrm{s}), 5.72\left(2 \mathrm{H}, \mathrm{s}, \mathrm{NH}_{2}\right), 6.80\left(2 \mathrm{H}^{\mathrm{ar}}, \mathrm{d}, J=8.7\right), 7.29$ $\left(2 \mathrm{H}^{\mathrm{ar}}, \mathrm{d}, J=8.7\right), 8.31(1 \mathrm{H}, \mathrm{s}, \mathrm{NH}) ;{ }^{13} \mathrm{C}$ NMR $\left(75 \mathrm{MHz}, d_{6}\right.$-DMSO) $\delta 55.1$ $\left(\mathrm{CH}_{3}\right), 113.9(2 \mathrm{CH}), 119.5(2 \mathrm{CH}), 133.7(\mathrm{Cq}), 154.0(\mathrm{Cq}), 156.3(\mathrm{Cq})$; IR (ATR) $v$ max: $3350(\mathrm{~N}-\mathrm{H}), 3317(\mathrm{~N}-\mathrm{H}), 2958,2928,2871,2855,1616$ $(\mathrm{C}=\mathrm{O}), 1574,1519,1478,1454,1289,1249,1226,1066$; MS $\left(\mathrm{ESI}^{+}\right) \mathrm{m} / \mathrm{z}$ $167\left([\mathrm{M}+\mathrm{H}]^{+}, 43\right), 189\left([\mathrm{M}+\mathrm{Na}]^{+}, 100\right), 205\left([\mathrm{M}+\mathrm{K}]^{+}, 13\right)$.

Procedure B: General procedure for the palladium-catalyzed reductive alkylation of primary ureas.

The monosubstituted urea $(1 \mathrm{mmol})$ and the aldehyde $(3 \mathrm{mmol})$ were dissolved in $10 \mathrm{~mL}$ of dry methanol in a $50 \mathrm{~mL}$ stainless steel autoclave, followed by the addition of $5 \%-\mathrm{Pd} / \mathrm{C}(53.2 \mathrm{mg}, 2.5 \mathrm{~mol}-\%)$. The reactor was tightly closed, purged three times and hydrogen pressure was introduced (5 bar). The reactor was then placed in a graphite bath on a magnetic stirrer and the reaction mixture was heated at $100{ }^{\circ} \mathrm{C}$ and stirred for 15 hours. After cooling to room temperature, hydrogen pressure was released and palladium $(\mathrm{Pd} / \mathrm{C})$ was filtered under vacuum over a Millipore filter and thoroughly washed with methanol several times. The filtrate was concentrated under reduced pressure. The corresponding alkylated ureas were purified either by recrystallization from diethyl ether or by column chromatography.

1-Benzyl-3-decylurea (16). The title compound was prepared from benzylurea 12 (150 mg, $1 \mathrm{mmol})$ and decanal $15(0.56 \mathrm{~mL}, 3 \mathrm{mmol})$ according to the procedure $\mathbf{B}$. The residue was purified by recrystallization from $\mathrm{Et}_{2} \mathrm{O}$ to give $\mathbf{1 6}(250 \mathrm{mg}, 86 \%$ yield $)$ as a white powder. m.p.: $96^{\circ} \mathrm{C}$; ${ }^{1} \mathrm{H}$ NMR (400 MHz, $d_{6}$-DMSO) $\delta 0.86(3 \mathrm{H}, \mathrm{t}, J=6.8), 1.18-1.31(14 \mathrm{H}, \mathrm{m})$, $1.31-1.40(2 \mathrm{H}, \mathrm{m}), 2.98$ (2H, dt app q, $J=6.5), 4.18(2 \mathrm{H}, \mathrm{d}, J=6.0), 5.90$ $(1 \mathrm{H}, \mathrm{t}, J=5.5, \mathrm{NH}), 6.26(1 \mathrm{H}, \mathrm{t}, J=6.0, \mathrm{NH}), 7.17-7.27\left(3 \mathrm{H}^{\mathrm{ar}}, \mathrm{m}\right), 7.27-$ $7.36\left(2 \mathrm{H}^{\mathrm{ar}}, \mathrm{m}\right) ;{ }^{13} \mathrm{C}$ NMR (100 MHz, $d_{6}$-DMSO) $\delta 14.0\left(\mathrm{CH}_{3}\right), 22.1\left(\mathrm{CH}_{2}\right)$, $26.4\left(\mathrm{CH}_{2}\right), 28.7\left(\mathrm{CH}_{2}\right), 28.8\left(\mathrm{CH}_{2}\right), 29.0\left(\mathrm{CH}_{2}\right), 29.1\left(\mathrm{CH}_{2}\right), 30.0\left(\mathrm{CH}_{2}\right)$, $31.3\left(\mathrm{CH}_{2}\right), 39.3\left(\mathrm{CH}_{2}\right), 42.9\left(\mathrm{CH}_{2}\right), 126.5(\mathrm{CH}), 127.0(2 \mathrm{CH}), 128.2(2$ $\mathrm{CH}), 141.1(\mathrm{Cq}), 158.1(\mathrm{Cq})$; IR (ATR) v max: $3320(\mathrm{~N}-\mathrm{H}), 2957,2920$, 2847, 1658 (C=O), 1625, 1596, 1564, 1499, 1479, 1464, 1443, 1315, 1298, 1246, 1228; $\mathrm{MS}\left(\mathrm{ESI}^{+}\right) \mathrm{m} / \mathrm{z} 291\left([\mathrm{M}+\mathrm{H}]^{+}, 12\right), 313\left([\mathrm{M}+\mathrm{Na}]^{+}, 100\right), 603$ $\left(\left[2 \mathrm{M}+\mathrm{Na}^{+}, 23\right)\right.$; HRMS $\left(\mathrm{ESI}^{+}\right)\left[\mathrm{M}+\mathrm{Na}^{+}\right.$for $\mathrm{C}_{18} \mathrm{H}_{30} \mathrm{~N}_{2} \mathrm{NaO}$ requires 313.2250, found 313.2249.

1-Decyl-3-hexylurea (17): The title compound was prepared from hexyl urea $8(144 \mathrm{mg}, 1 \mathrm{mmol})$ and decanal $15(0.56 \mathrm{~mL}, 3 \mathrm{mmol})$ according to the procedure $\mathbf{B}$. The residue was purified by recrystallization from $\mathrm{Et}_{2} \mathrm{O}$ to give $\mathbf{1 7}\left(250 \mathrm{mg}, 88 \%\right.$ yield) as a white powder. m.p.: $75{ }^{\circ} \mathrm{C}$; ${ }^{1} \mathrm{H}$ NMR (400 MHz, $d_{6}$-DMSO) $\delta 0.85(6 \mathrm{H}, \mathrm{t}, J=6.4), 1.17-1.38(24 \mathrm{H}, \mathrm{m}), 2.94(4 \mathrm{H}, \mathrm{dt}$ app q, $J=6.2), 5.73(2 \mathrm{H}, \mathrm{t}, J=5.4,2 \mathrm{NH}) ;{ }^{13} \mathrm{C} \mathrm{NMR}\left(100 \mathrm{MHz}, \mathrm{CD}_{3} \mathrm{OD}\right) \delta$ $14.4\left(\mathrm{CH}_{3}\right), 14.5\left(\mathrm{CH}_{3}\right), 23.7\left(\mathrm{CH}_{2}\right), 23.8\left(\mathrm{CH}_{2}\right), 27.7\left(\mathrm{CH}_{2}\right), 28.0\left(\mathrm{CH}_{2}\right)$, $30.48\left(\mathrm{CH}_{2}\right), 30.52\left(\mathrm{CH}_{2}\right), 30.7\left(\mathrm{CH}_{2}\right), 30.8\left(\mathrm{CH}_{2}\right), 31.36\left(\mathrm{CH}_{2}\right), 31.39$ $\left(\mathrm{CH}_{2}\right), 32.8\left(\mathrm{CH}_{2}\right), 33.1\left(\mathrm{CH}_{2}\right), 41.0\left(2 \mathrm{CH}_{2}\right), 161.4(\mathrm{Cq})$; IR (ATR) v max: $3326(\mathrm{~N}-\mathrm{H}), 2956,2920,2849,1610$ (C=O), 1572, 1477, 1461, 1250, 1223 , 725; $\mathrm{MS}\left(\mathrm{ESI}^{+}\right) \mathrm{m} / \mathrm{z} 285\left([\mathrm{M}+\mathrm{H}]^{+}, 67\right), 307\left([\mathrm{M}+\mathrm{Na}]^{+}, 100\right), 591([2 \mathrm{M}+$ $\mathrm{Na}]^{+}, 73$ ); HRMS $\left(\mathrm{ESI}^{+}\right)[\mathrm{M}+\mathrm{H}]^{+}$for $\mathrm{C}_{17} \mathrm{H}_{37} \mathrm{~N}_{2} \mathrm{O}$ requires 285.2900, found 285.2902

1-Dodecyl-3-hexylurea (25). The title compound was prepared from hexyl urea 8 (144 mg, $1 \mathrm{mmol})$ and lauric aldehyde $\mathbf{1 8}(0.66 \mathrm{~mL}, 3 \mathrm{mmol})$ according to the procedure $\mathbf{B}$. The residue was purified by recrystallization from $\mathrm{Et}_{2} \mathrm{O}$ to give $25(270 \mathrm{mg}, 86 \%$ yield $)$ as a white powder. m.p.: $82{ }^{\circ} \mathrm{C}$; ${ }^{1} \mathrm{H}$ NMR (300 MHz, $d_{6}$-DMSO) $\delta 0.85$ (6H, t, $\left.J=6.3\right), 1.16-1.40(28 \mathrm{H}, \mathrm{m})$, $2.94(4 \mathrm{H}$, dt app q, $J=6.2), 5.70(2 \mathrm{H}, \mathrm{t}, J=5.6,2 \mathrm{NH}) ;{ }^{13} \mathrm{C} \mathrm{NMR}(100$ $\left.\mathrm{MHz}, \mathrm{CD}_{3} \mathrm{OD}\right) \delta 14.38\left(\mathrm{CH}_{3}\right), 14.44\left(\mathrm{CH}_{3}\right), 23.70\left(\mathrm{CH}_{2}\right), 23.74\left(\mathrm{CH}_{2}\right), 27.7$ $\left(\mathrm{CH}_{2}\right), 28.0\left(\mathrm{CH}_{2}\right), 30.50\left(\mathrm{CH}_{2}\right), 30.51\left(\mathrm{CH}_{2}\right), 30.74\left(\mathrm{CH}_{2}\right), 30.75\left(\mathrm{CH}_{2}\right)$, $30.77\left(\mathrm{CH}_{2}\right), 30.80\left(\mathrm{CH}_{2}\right), 31.36\left(\mathrm{CH}_{2}\right), 31.38\left(\mathrm{CH}_{2}\right), 32.8\left(\mathrm{CH}_{2}\right), 33.1$ $\left(\mathrm{CH}_{2}\right), 41.0\left(2 \mathrm{CH}_{2}\right), 161.4(\mathrm{Cq})$; IR (ATR) v max: $3328(\mathrm{~N}-\mathrm{H}), 2955,2920$, 2848, $1611(\mathrm{C}=\mathrm{O}), 1571,1476,1463,1263,1245,723$; MS (ESI $\left.{ }^{+}\right) \mathrm{m} / z 313$ 
$\left([\mathrm{M}+\mathrm{H}]^{+}, 100\right), 335\left([\mathrm{M}+\mathrm{Na}]^{+}, 17\right) ; \operatorname{HRMS}\left(\mathrm{ESI}^{+}\right)[\mathrm{M}+\mathrm{H}]^{+}$for $\mathrm{C}_{19} \mathrm{H}_{41} \mathrm{~N}_{2} \mathrm{O}$ requires 313.3213 , found 313.3212 .

1-Hexyl-3-octylurea (26). The title compound was prepared from hexyl urea $8(144 \mathrm{mg}, 1 \mathrm{mmol})$ and octanal $19(0.47 \mathrm{~mL}, 3 \mathrm{mmol})$ according to the procedure $\mathbf{B}$. The residue was purified by recrystallization from $\mathrm{Et}_{2} \mathrm{O}$ to give 26 (210 mg, 82\% yield) as a white powder. m.p.: $70{ }^{\circ} \mathrm{C} ;{ }^{1} \mathrm{H}$ NMR $(500$ MHz, $d_{6}$-DMSO) $\delta 0.86(6 \mathrm{H}, \mathrm{t}, J=7.0), 1.17-1.30(16 \mathrm{H}, \mathrm{m}), 1.30-1.40$ $(4 \mathrm{H}, \mathrm{m}), 2.94(4 \mathrm{H}, \mathrm{dt}$ app q, $J=6.5), 5.70(2 \mathrm{H}, \mathrm{t}, J=5.4,2 \mathrm{NH}) ;{ }^{13} \mathrm{C} \mathrm{NMR}$ (125 MHz, $d_{6}$-DMSO) $\delta 13.91\left(\mathrm{CH}_{3}\right), 13.95\left(\mathrm{CH}_{3}\right), 22.1\left(2 \mathrm{CH}_{2}\right), 26.1$ $\left(\mathrm{CH}_{2}\right), 26.4\left(\mathrm{CH}_{2}\right), 28.7\left(\mathrm{CH}_{2}\right), 28.8\left(\mathrm{CH}_{2}\right), 30.0\left(\mathrm{CH}_{2}\right), 30.1\left(\mathrm{CH}_{2}\right), 31.1$ $\left(\mathrm{CH}_{2}\right), 31.2\left(\mathrm{CH}_{2}\right), 39.2\left(2 \mathrm{CH}_{2}\right), 158.1(\mathrm{Cq})$; IR (ATR) $v$ max: $3324(\mathrm{~N}-\mathrm{H})$ 2956, 2923, 2869, 2851, 1612 (C=O), 1574, 1477, 1461, 1290, 1264, 1245, 1226, 1212, 1077, 727; MS $\left(\mathrm{ESI}^{+}\right) \mathrm{m} / z 257\left([\mathrm{M}+\mathrm{H}]^{+}, 100\right), 279\left([\mathrm{M}+\mathrm{Na}]^{+}\right.$ 22); HRMS $\left(\mathrm{ESI}^{+}\right)[\mathrm{M}+\mathrm{H}]^{+}$for $\mathrm{C}_{15} \mathrm{H}_{33} \mathrm{~N}_{2} \mathrm{O}$ requires 257.2587, found 257.2585

1,3-Dihexylurea (27). The title compound was prepared from hexyl urea 8 $(144 \mathrm{mg}, 1 \mathrm{mmol})$ and hexanal $20(0.37 \mathrm{~mL}, 3 \mathrm{mmol})$ according to the procedure $\mathbf{B}$. The residue was purified by recrystallization from $\mathrm{Et}_{2} \mathrm{O}$ by to give 27 (182 mg, 80\% yield) as a white powder. m.p.: $87{ }^{\circ} \mathrm{C}$; ${ }^{1} \mathrm{H}$ NMR $(400$ $\mathrm{MHz}, d_{6}$-DMSO) $\delta 0.86(6 \mathrm{H}, \mathrm{t}, J=6.8), 1.15-1.42(16 \mathrm{H}, \mathrm{m}), 2.94(4 \mathrm{H}, \mathrm{dt}$ app q, $J=6.5), 5.71(2 \mathrm{H}, \mathrm{t}, J=5.7,2 \mathrm{NH}) ;{ }^{13} \mathrm{C} \mathrm{NMR}\left(100 \mathrm{MHz}, d_{6^{-}}\right.$ DMSO) $\delta 13.9\left(2 \mathrm{CH}_{3}\right), 22.1\left(2 \mathrm{CH}_{2}\right), 26.1\left(2 \mathrm{CH}_{2}\right), 30.0\left(2 \mathrm{CH}_{2}\right), 31.1(2$ $\left.\mathrm{CH}_{2}\right), 39.2\left(2 \mathrm{CH}_{2}\right), 158.1(\mathrm{Cq})$; IR (ATR) v max: $3324(\mathrm{~N}-\mathrm{H}), 2956,2929$, 2856, $1611(\mathrm{C}=\mathrm{O}), 1574,1477,1461,1299,1249,1220,1076,728$; MS $\left(\mathrm{ESI}^{+}\right) \mathrm{m} / \mathrm{z} 229\left([\mathrm{M}+\mathrm{H}]^{+}, 100\right), 251\left([\mathrm{M}+\mathrm{Na}]^{+}, 34\right) ; \mathrm{HRMS}\left(\mathrm{ESI}^{+}\right)[\mathrm{M}+$ $\mathrm{H}]^{+}$for $\mathrm{C}_{13} \mathrm{H}_{29} \mathrm{~N}_{2} \mathrm{O}$ requires 229.2274, found 229.2272.

1-Hexyl-3-pentylurea (28). The title compound was prepared from hexyl urea 8 (144 mg, $1 \mathrm{mmol})$ and valeraldehyde $21(0.32 \mathrm{~mL}, 3 \mathrm{mmol})$ according to the procedure $\mathbf{B}$. The residue was purified by column chromatography (EtOAc/cyclohexane 40:60) to give 28 (178 mg, 78\% yield) as a yellowish powder. m.p.: $76-78{ }^{\circ} \mathrm{C} ;{ }^{1} \mathrm{H}$ NMR $\left(400 \mathrm{MHz}, d_{6-}\right.$ DMSO): $\delta 0.86$ (6H, t, $J=7.0), 1.18-1.41(14 \mathrm{H}, \mathrm{m}), 2.94$ (4H, dt app q, $J=$ 6.5), $5.71(2 \mathrm{H}, \mathrm{t}, J=5.5,2 \mathrm{NH}) ;{ }^{13} \mathrm{C}$ NMR (100 MHz, $d_{6}$-DMSO): $\delta 13.93$ $\left(\mathrm{CH}_{3}\right), 13.97\left(\mathrm{CH}_{3}\right), 21.9\left(\mathrm{CH}_{2}\right), 22.1\left(\mathrm{CH}_{2}\right), 26.1\left(\mathrm{CH}_{2}\right), 28.6\left(\mathrm{CH}_{2}\right), 29.7$ $\left(\mathrm{CH}_{2}\right), 30.0\left(\mathrm{CH}_{2}\right), 31.1\left(\mathrm{CH}_{2}\right), 39.18\left(\mathrm{CH}_{2}\right), 39.22\left(\mathrm{CH}_{2}\right), 158.1(\mathrm{Cq}) ; \mathrm{IR}$ (ATR) $v$ max: $3327(\mathrm{~N}-\mathrm{H}), 2955,2929,2857,1618(\mathrm{C}=\mathrm{O}), 1574,1479$, 1446, 1426, 1259, 1074; $\mathrm{MS}\left(\mathrm{ESI}^{+}\right) \mathrm{m} / z .158\left(\left[\mathrm{M}+\mathrm{H}-\mathrm{C}_{4} \mathrm{H}_{9}\right]^{+}, 38\right), 172$ $\left(\left[\mathrm{M}+\mathrm{H}-\mathrm{C}_{3} \mathrm{H}_{7}\right]^{+}, 35\right), 215\left([\mathrm{M}+\mathrm{H}]^{+}, 100\right), 237\left([\mathrm{M}+\mathrm{Na}]^{+}, 12\right) ; \mathrm{HRMS}$ $\left(\mathrm{ESI}^{+}\right)[\mathrm{M}+\mathrm{H}]^{+}$for $\mathrm{C}_{12} \mathrm{H}_{27} \mathrm{~N}_{2} \mathrm{O}$ requires 215.2118, found 215.2117.

1-Butyl-3-hexylurea (29). The title compound was prepared from hexyl urea $8(144 \mathrm{mg}, 1 \mathrm{mmol})$ and butyraldehyde $22(0.27 \mathrm{~mL}, 3 \mathrm{mmol})$ according to the procedure $\mathbf{B}$. The residue was purified by column chromatography (EtOAc/cyclohexane 20:80) to give 29 (130 mg, 65\% yield) as a white powder. m.p.: $70-75{ }^{\circ} \mathrm{C} ;{ }^{1} \mathrm{H}$ NMR $\left(300 \mathrm{MHz}, d_{6}\right.$-DMSO): $\delta 0.86(6 \mathrm{H}, \mathrm{t}, J=7.0), 1.18-1.40(12 \mathrm{H}, \mathrm{m}), 2.90-3.00(4 \mathrm{H}, \mathrm{m}), 5.70(2 \mathrm{H}, \mathrm{t}$, $J=5.5,2 \mathrm{NH}) ;{ }^{13} \mathrm{C}$ NMR (125 MHz, $d_{6}$-DMSO): $\delta 13.7\left(\mathrm{CH}_{3}\right), 13.9\left(\mathrm{CH}_{3}\right)$, $19.5\left(\mathrm{CH}_{2}\right), 22.1\left(\mathrm{CH}_{2}\right), 26.1\left(\mathrm{CH}_{2}\right), 30.0\left(\mathrm{CH}_{2}\right), 31.0\left(\mathrm{CH}_{2}\right), 32.2\left(\mathrm{CH}_{2}\right)$, $38.9\left(\mathrm{CH}_{2}\right), 39.2\left(\mathrm{CH}_{2}\right), 158.1(\mathrm{Cq})$; IR (ATR) v max: $3324(\mathrm{~N}-\mathrm{H}), 2954$, 2929, 2857, 1616 (C=O), 1569, 1479, 1464, 1272, 1245, 1225, 1075; MS $\left(\mathrm{ESI}^{+}\right) \mathrm{m} / \mathrm{z} 201\left([\mathrm{M}+\mathrm{H}]^{+}, 38\right), 223\left([\mathrm{M}+\mathrm{Na}]^{+}, 100\right), 423\left([2 \mathrm{M}+\mathrm{Na}]^{+}, 35\right)$; HRMS $\left(\mathrm{ESI}^{+}\right)[\mathrm{M}+\mathrm{Na}]^{+}$for $\mathrm{C}_{11} \mathrm{H}_{24} \mathrm{~N}_{2} \mathrm{NaO}$ requires 223.1781, found 223.1782 .

1-Benzyl-3-hexylurea (30). The title compound was prepared from hexyl urea 8 (144 mg, $1 \mathrm{mmol})$ and benzaldehyde $23(0.68 \mathrm{~mL}, 3 \mathrm{mmol})$ according to the procedure $\mathbf{B}$. The residue was purified by column chromatography (EtOAc/cyclohexane 20:80) to give 30 (218 mg, 93\% yield) as a white powder. m.p.: $74{ }^{\circ} \mathrm{C} ;{ }^{1} \mathrm{H}$ NMR (300 MHz, $d_{6}$-DMSO) $\delta$ 0.86 (3H, t, $J=6.7$ ), 1.20-1.42 (8H, m), 2.99 (2H, dt app q, $J=6.4), 4.18$
$(2 \mathrm{H}, \mathrm{d}, J=6.0), 5.88(1 \mathrm{H}, \mathrm{t}, J=6.3, \mathrm{NH}), 6.24(1 \mathrm{H}, \mathrm{t}, J=5.1, \mathrm{NH}), 7.16-$ $7.26\left(3 \mathrm{H}^{\mathrm{ar}}, \mathrm{m}\right), 7.26-7.35\left(2 \mathrm{H}^{\mathrm{ar}}, \mathrm{m}\right) ;{ }^{13} \mathrm{C}$ NMR $\left(125 \mathrm{MHz}, d_{6}\right.$-DMSO) $\delta 13.9$ $\left(\mathrm{CH}_{3}\right), 22.1\left(\mathrm{CH}_{2}\right), 26.0\left(\mathrm{CH}_{2}\right), 30.0\left(\mathrm{CH}_{2}\right), 31.0\left(\mathrm{CH}_{2}\right), 39.3\left(\mathrm{CH}_{2}\right), 42.9$ $\left(\mathrm{CH}_{2}\right), 126.5(\mathrm{CH}), 127.0(2 \mathrm{CH}), 128.2(2 \mathrm{CH}), 141.0(\mathrm{Cq}), 158.0(\mathrm{Cq})$; IR (ATR) $v$ max: $3350(\mathrm{~N}-\mathrm{H}), 3318(\mathrm{~N}-\mathrm{H}), 3033,2958,2928,2855,1617$ $(\mathrm{C}=\mathrm{O}), 1575,1493,1478,1454,1289,1249,1226,1081,1065,1025$; MS $\left(\mathrm{ESI}^{+}\right) \mathrm{m} / \mathrm{z} 257\left([\mathrm{M}+\mathrm{Na}]^{+}, 100\right), 491\left(\left[2 \mathrm{M}+\mathrm{Na}^{+}, 7\right) ; \mathrm{HRMS}\left(\mathrm{ESI}^{+}\right)[\mathrm{M}+\right.$ $\mathrm{Na}^{+}$for $\mathrm{C}_{14} \mathrm{H}_{22} \mathrm{~N}_{2} \mathrm{NaO}$ requires 257.1624, found 257.1626.

1-Hexyl-3-(3-phenylpropyl)urea (31). The title compound was prepared from hexyl urea 8 (144 mg, $1 \mathrm{mmol})$ and 3-phenylpropionaldehyde $\mathbf{2 4}(0.40$ $\mathrm{mL}, 3 \mathrm{mmol}$ ) according to the procedure $\mathbf{B}$. The residue was purified by column chromatography (EtOAc/cyclohexane 20:80) to give 31 (235 mg, $90 \%$ yield) as a white powder. m.p.: $78{ }^{\circ} \mathrm{C} ;{ }^{1} \mathrm{H}$ NMR $\left(300 \mathrm{MHz}, d_{6}\right.$-DMSO) $\delta 0.85(3 \mathrm{H}, \mathrm{t}, J=6.7), 1.17-1.42(8 \mathrm{H}, \mathrm{m}), 1.64$ ( $2 \mathrm{H}$, quintet, $J=7.0), 2.56$ $(2 \mathrm{H}, \mathrm{t}, J=7.3), 2.95$ ( $4 \mathrm{H}$, app quintet, $J=6.4), 5.74(1 \mathrm{H}, \mathrm{t}, J=5.6, \mathrm{NH})$, $5.81(1 \mathrm{H}, \mathrm{t}, J=5.6, \mathrm{NH}), 7.12-7.23\left(3 \mathrm{H}^{\mathrm{ar}}, \mathrm{m}\right), 7.23-7.33\left(2 \mathrm{H}^{\mathrm{ar}}, \mathrm{m}\right) ;{ }^{13} \mathrm{C}$ NMR (125 MHz, $d_{6}$-DMSO) $\delta 13.9\left(\mathrm{CH}_{3}\right), 22.1\left(\mathrm{CH}_{2}\right), 26.1\left(\mathrm{CH}_{2}\right), 30.0$ $\left(\mathrm{CH}_{2}\right), 31.0\left(\mathrm{CH}_{2}\right), 31.9\left(\mathrm{CH}_{2}\right), 32.5\left(\mathrm{CH}_{2}\right), 38.8\left(\mathrm{CH}_{2}\right), 39.2\left(\mathrm{CH}_{2}\right), 125.7$ $(\mathrm{CH}), 128.2(4 \mathrm{CH}), 141.8(\mathrm{Cq}), 158.1(\mathrm{Cq})$; IR (ATR) v max: $3328(\mathrm{~N}-\mathrm{H})$, 2951, 2924, 2855, 1621 (C=O), 1583, 1496, 1459, 1378, 1269, 1063, 1029; MS $\left(\mathrm{ESI}^{+}\right) \mathrm{m} / z 263\left([\mathrm{M}+\mathrm{H}]^{+}, 21\right), 285\left([\mathrm{M}+\mathrm{Na}]^{+}, 100\right), 547\left(\left[2 \mathrm{M}+\mathrm{Na}^{+}\right.\right.$, 5); $\mathrm{HRMS}\left(\mathrm{ESI}^{+}\right)[\mathrm{M}+\mathrm{Na}]^{+}$for $\mathrm{C}_{16} \mathrm{H}_{26} \mathrm{~N}_{2} \mathrm{NaO}$ requires 285.1937, found 285.1939 .

1-Decyl-3-methylurea (33). The title compound was prepared from methyl urea 32 (74 mg, $1 \mathrm{mmol})$ and decanal $15(0.66 \mathrm{~mL}, 3 \mathrm{mmol})$ according to the procedure $\mathbf{B}$. The residue was purified by recrystallization from $\mathrm{Et}_{2} \mathrm{O}$ to give 33 (128 mg, 60\% yield) as a white powder. m.p.: $83{ }^{\circ} \mathrm{C}$; ${ }^{1} \mathrm{H}$ NMR (300 $\mathrm{MHz}, d_{6}$-DMSO) $\delta 0.85(3 \mathrm{H}, \mathrm{t}, J=6.7), 1.11-1.40(16 \mathrm{H}, \mathrm{m}), 2.51(3 \mathrm{H}$, masked d, $J=4.5), 2.93$ (2H, dt app q, $J=6.4), 5.61(1 \mathrm{H}, \mathrm{d}, J=4.4, \mathrm{NH})$, $5.79(1 \mathrm{H}, \mathrm{t}, J=5.3, \mathrm{NH}) ;{ }^{13} \mathrm{C} \mathrm{NMR}\left(100 \mathrm{MHz}, d_{6}\right.$-DMSO) $\delta 14.0\left(\mathrm{CH}_{3}\right)$, $22.1\left(\mathrm{CH}_{2}\right), 26.36\left(\mathrm{CH}_{3}\right), 26.40\left(\mathrm{CH}_{2}\right), 28.7\left(\mathrm{CH}_{2}\right), 28.8\left(\mathrm{CH}_{2}\right), 29.0\left(\mathrm{CH}_{2}\right)$, $29.1\left(\mathrm{CH}_{2}\right), 30.1\left(\mathrm{CH}_{2}\right), 31.3\left(\mathrm{CH}_{2}\right), 39.3\left(\mathrm{CH}_{2}\right), 158.7(\mathrm{Cq})$; IR (ATR) v max: $3334(\mathrm{~N}-\mathrm{H}), 2954,2921,2849,1617$ (C=O), 1579, 1521, 1479, 1466, 1417, 1311, 1282, 1254, 1237, 1068; MS $\left(\mathrm{ESI}^{+}\right) \mathrm{m} / z 215\left([\mathrm{M}+\mathrm{H}]^{+}, 100\right)$, $238\left([\mathrm{M}+\mathrm{Na}]^{+}, 54\right), 451\left([2 \mathrm{M}+\mathrm{Na}]^{+}, 14\right) ; \mathrm{HRMS}\left(\mathrm{ESI}^{+}\right)[\mathrm{M}+\mathrm{H}]^{+}$for $\mathrm{C}_{12} \mathrm{H}_{27} \mathrm{~N}_{2} \mathrm{O}$ requires 215.2118, found 215.2121.

1-Cyclohexyl-3-decylurea (34). The title compound was prepared from 1cyclohexylurea 9 (142 mg, $1 \mathrm{mmol})$ and decanal $15(0.56 \mathrm{~mL}, 3 \mathrm{mmol})$ according to the procedure $\mathbf{B}$. The residue was purified by recrystallization from $\mathrm{Et}_{2} \mathrm{O}$ to give 34 (220 mg, 78\% yield) as a white powder. m.p.: $89{ }^{\circ} \mathrm{C}$; ${ }^{1} \mathrm{H}$ NMR (300 MHz, $d_{6}$-DMSO) $\delta 0.85(3 \mathrm{H}, \mathrm{t}, J=6.6), 0.96-1.16(3 \mathrm{H}, \mathrm{m})$, $1.16-1.40(18 \mathrm{H}, \mathrm{m}), 1.42-1.56(1 \mathrm{H}, \mathrm{m}), 1.56-1.77(4 \mathrm{H}, \mathrm{m}), 2.93(2 \mathrm{H}, \mathrm{dt}$ app q, $J=6.4), 3.25-3.38(1 \mathrm{H}, \mathrm{m}), 5.62(2 \mathrm{H}$, app d, $J=7.4,2 \mathrm{NH}) ;{ }^{13} \mathrm{C}$ NMR $\left(100 \mathrm{MHz}, \mathrm{CD}_{3} \mathrm{OD}\right) \delta 14.5\left(\mathrm{CH}_{3}\right), 23.7\left(\mathrm{CH}_{2}\right), 26.1\left(2 \mathrm{CH}_{2}\right), 26.8$ $\left(\mathrm{CH}_{2}\right), 28.0\left(\mathrm{CH}_{2}\right), 30.47\left(\mathrm{CH}_{2}\right), 30.50\left(\mathrm{CH}_{2}\right), 30.7\left(\mathrm{CH}_{2}\right), 30.8\left(\mathrm{CH}_{2}\right), 31.4$ $\left(\mathrm{CH}_{2}\right), 33.1\left(\mathrm{CH}_{2}\right), 34.8\left(2 \mathrm{CH}_{2}\right), 40.9\left(\mathrm{CH}_{2}\right), 49.8(\mathrm{CH}), 160.6(\mathrm{Cq})$; IR (ATR) $v$ max: $3347(\mathrm{~N}-\mathrm{H}), 3312(\mathrm{~N}-\mathrm{H}), 2961,2922,2850,1616(\mathrm{C}=\mathrm{O})$, 1589, 1579, 1521, 1461, 1279, 1252, 1236; MS (ESI $\left.{ }^{+}\right) \mathrm{m} / z 283\left([\mathrm{M}+\mathrm{H}]^{+}\right.$, 100), $565\left([2 \mathrm{M}+\mathrm{H}]^{+}, 21\right)$; HRMS $\left(\mathrm{ESI}^{+}\right)[\mathrm{M}+\mathrm{H}]^{+}$for $\mathrm{C}_{17} \mathrm{H}_{35} \mathrm{~N}_{2} \mathrm{O}$ requires 283.2744, found 283.2738 .

1-(Cyclohexylmethyl)-3-decylurea (35). The title compound was prepared from 1-(cyclohexylmethyl) urea $\mathbf{1 0}(156 \mathrm{mg}, 1 \mathrm{mmol})$ and decanal $\mathbf{1 5}(0.56$ $\mathrm{mL}, 3 \mathrm{mmol}$ ) according to the procedure $\mathbf{B}$. The residue was purified by recrystallization from $\mathrm{Et}_{2} \mathrm{O}$ to give $\mathbf{3 5}$ (236 $\mathrm{mg}, 80 \%$ yield) as a white powder. m.p.: $82{ }^{\circ} \mathrm{C} ;{ }^{1} \mathrm{H}$ NMR ( $300 \mathrm{MHz}, d_{6}$-DMSO) $\delta 0.85(3 \mathrm{H}, \mathrm{t}, J=6.6$ ), $1.05-1.40(22 \mathrm{H}, \mathrm{m}), 1.52-1.72(5 \mathrm{H}, \mathrm{m}), 2.80(2 \mathrm{H}, \mathrm{t}, J=6.4), 2.94(2 \mathrm{H}, \mathrm{dt}$ app q, $J=6.4), 5.68(1 \mathrm{H}, \mathrm{t}, J=5.3, \mathrm{NH}), 5.75(1 \mathrm{H}, \mathrm{t}, J=5.7, \mathrm{NH}) ;{ }^{13} \mathrm{C}$ NMR (100 MHz, CD $3 \mathrm{OD}) \delta 14.5\left(\mathrm{CH}_{3}\right), 23.7\left(\mathrm{CH}_{2}\right), 27.1\left(2 \mathrm{CH}_{2}\right), 27.7$ $\left(\mathrm{CH}_{2}\right), 28.0\left(\mathrm{CH}_{2}\right), 30.48\left(\mathrm{CH}_{2}\right), 30.52\left(\mathrm{CH}_{2}\right), 30.7\left(\mathrm{CH}_{2}\right), 30.8\left(\mathrm{CH}_{2}\right), 31.4$ 
$\left(\mathrm{CH}_{2}\right), 31.9\left(2 \mathrm{CH}_{2}\right), 33.1\left(\mathrm{CH}_{2}\right), 39.9(\mathrm{CH}), 41.0\left(\mathrm{CH}_{2}\right), 47.4\left(\mathrm{CH}_{2}\right), 161.4$ (Cq); IR (ATR) $v$ max: $3343(\mathrm{~N}-\mathrm{H}), 3306(\mathrm{~N}-\mathrm{H}), 2923,2850,1619(\mathrm{C}=\mathrm{O})$, 1577, 1522, 1480, 1460, 1251, 1234; MS (ESI $\left.{ }^{+}\right) \mathrm{m} / \mathrm{z} 297\left([\mathrm{M}+\mathrm{H}]^{+}, 56\right)$, $319\left(\left[\mathrm{M}+\mathrm{Na}^{+}, 100\right), 615\left([2 \mathrm{M}+\mathrm{Na}]^{+}, 61\right) ; \mathrm{HRMS}\left(\mathrm{ESI}^{+}\right)[\mathrm{M}+\mathrm{H}]^{+}\right.$for $\mathrm{C}_{18} \mathrm{H}_{37} \mathrm{~N}_{2} \mathrm{O}$ requires 297.2900, found 297.2899.

1-Decyl-3-(2-phenylethyl)urea (36). The title compound was prepared from 1-(2-phenylethyl)urea $11(164 \mathrm{mg}, 1 \mathrm{mmol})$ and decanal $15(0.56 \mathrm{~mL}$, $3 \mathrm{mmol}$ ) according to the procedure $\mathbf{B}$. The residue was purified by recrystallization from $\mathrm{Et}_{2} \mathrm{O}$ to give $\mathbf{3 6}(239 \mathrm{mg}, 79 \%$ yield) as a white powder. m.p.: $92{ }^{\circ} \mathrm{C} ;{ }^{1} \mathrm{H} \mathrm{NMR}\left(300 \mathrm{MHz}, d_{6}\right.$-DMSO) $\delta 0.85$ (3H, t, $J=6.4$ ) $1.10-1.41(16 \mathrm{H}, \mathrm{m}), 2.64(2 \mathrm{H}, \mathrm{t}, J=7.3), 2.94(2 \mathrm{H}, \mathrm{q}, J=6.3), 3.20(2 \mathrm{H}, \mathrm{q}$, $J=6.7), 5.74(1 \mathrm{H}, \mathrm{t}, J=5.6, \mathrm{NH}), 5.82(1 \mathrm{H}, \mathrm{t}, J=5.3, \mathrm{NH}), 7.15-7.24(3 \mathrm{H}$, m), 7.25-7.35 (2H, m); ${ }^{13} \mathrm{C}$ NMR (100 MHz, $d_{6}$-DMSO) $\delta 14.0\left(\mathrm{CH}_{3}\right), 22.1$ $\left(\mathrm{CH}_{2}\right), 26.4\left(\mathrm{CH}_{2}\right), 28.7\left(\mathrm{CH}_{2}\right), 28.8\left(\mathrm{CH}_{2}\right), 29.0\left(\mathrm{CH}_{2}\right), 29.1\left(\mathrm{CH}_{2}\right), 30.0$ $\left(\mathrm{CH}_{2}\right), 31.3\left(\mathrm{CH}_{2}\right), 36.2\left(\mathrm{CH}_{2}\right), 39.2\left(\mathrm{CH}_{2}\right), 40.9\left(\mathrm{CH}_{2}\right), 126.0(\mathrm{CH}), 128.3$ $(2 \mathrm{CH}), 128.7(2 \mathrm{CH}), 139.8(\mathrm{Cq}), 158.0(\mathrm{Cq})$; IR (ATR) $\vee$ max: $3310(\mathrm{~N}-$ H), 2957, 2920, 2871, 2848, 1613 (C=O), 1597, 1566, 1500, 1478, 1463, 1444, 1315, 1299, 1247, 1230; MS $\left(\mathrm{ESI}^{+}\right) \mathrm{m} / z 305\left([\mathrm{M}+\mathrm{H}]^{+}, 26\right), 327([\mathrm{M}$ $\left.+\mathrm{Na}]^{+}, 100\right), 631\left(\left[2 \mathrm{M}+\mathrm{Na}^{+}, 17\right) ; \mathrm{HRMS}\left(\mathrm{ESI}^{+}\right)[\mathrm{M}+\mathrm{H}]^{+}\right.$for $\mathrm{C}_{19} \mathrm{H}_{33} \mathrm{~N}_{2} \mathrm{O}$ requires 305.2587, found $305.2593(-1.9 \mathrm{ppm}) ;\left[\mathrm{M}+\mathrm{Na}^{+}\right.$for $\mathrm{C}_{19} \mathrm{H}_{32} \mathrm{~N}_{2} \mathrm{NaO}$ requires 327.2407, found 327.2408.

1-Decyl-3-(1-phenylethyl)urea (37). The title compound was prepared from 1-phenylethylurea 13 (164 mg, $1 \mathrm{mmol})$ and decanal $15(0.56 \mathrm{~mL}, 3$ $\mathrm{mmol}$ ) according to the procedure $\mathbf{B}$. The residue was purified by column chromatography (EtOAc/cyclohexane 20:80) to give 37 (218 mg, 72\% yield) as a white powder. m.p.: $65{ }^{\circ} \mathrm{C} ;{ }^{1} \mathrm{H}$ NMR $\left(400 \mathrm{MHz}, d_{6}\right.$-DMSO) $\delta$ 0.85 (3H, t, $J=6.7), 1.10-1.40(16 \mathrm{H}, \mathrm{m}), 1.29(3 \mathrm{H}, \mathrm{d}, J=6.8), 2.94(2 \mathrm{H}$, $\mathrm{m}), 4.71(1 \mathrm{H}$, dq app quintet, $J=7.2), 5.74(1 \mathrm{H}, \mathrm{t}, J=5.5, \mathrm{NH}), 6.22(1 \mathrm{H}, \mathrm{d}$ $J=8.1, \mathrm{NH}), 7.20\left(1 \mathrm{H}^{\mathrm{ar}}, \mathrm{tt}, J=6.0,1.8\right), 7.25-7.34\left(4 \mathrm{H}^{\mathrm{ar}}, \mathrm{m}\right) ;{ }^{13} \mathrm{C} \mathrm{NMR}$ $\left(100 \mathrm{MHz}, d_{6}\right.$-DMSO) $\delta 14.0\left(\mathrm{CH}_{3}\right), 22.1\left(\mathrm{CH}_{2}\right), 23.4\left(\mathrm{CH}_{3}\right), 26.4\left(\mathrm{CH}_{2}\right)$, $28.7\left(\mathrm{CH}_{2}\right), 28.8\left(\mathrm{CH}_{2}\right), 29.0\left(\mathrm{CH}_{2}\right), 29.1\left(\mathrm{CH}_{2}\right), 30.0\left(\mathrm{CH}_{2}\right), 31.3\left(\mathrm{CH}_{2}\right)$, $39.1\left(\mathrm{CH}_{2}\right), 48.5(\mathrm{CH}), 125.7(2 \mathrm{CH}), 126.4(\mathrm{CH}), 128.1(2 \mathrm{CH}), 145.9(\mathrm{Cq})$ $157.3(\mathrm{Cq})$; IR (ATR) $v$ max: 3347 (N-H), $3311(\mathrm{~N}-\mathrm{H}), 2961,2923,2902$, 2851, $1614(\mathrm{C}=\mathrm{O}), 1591,1581,1523,1481,1461,1447,1251,1083,1069$; MS $\left(\mathrm{ESI}^{+}\right) \mathrm{m} / \mathrm{z} 305\left([\mathrm{M}+\mathrm{H}]^{+}, 100\right), 328\left([\mathrm{M}+\mathrm{Na}]^{+}, 59\right), 609\left([2 \mathrm{M}+\mathrm{H}]^{+}\right.$, 24), $631\left([2 \mathrm{M}+\mathrm{Na}]^{+}, 24\right) ; \mathrm{HRMS}\left(\mathrm{ESI}^{+}\right)[\mathrm{M}+\mathrm{H}]^{+}$for $\mathrm{C}_{19} \mathrm{H}_{33} \mathrm{~N}_{2} \mathrm{O}$ requires 305.2587 , found 305.2582 .

\section{1-Decyl-3-(4-methoxy-phenyl)-urea (38).}

The title compound was prepared from 4-methoxy-phenylurea 14 (306 mg, $1 \mathrm{mmol})$ and decanal $15(0.56 \mathrm{~mL}, 3 \mathrm{mmol})$ according to the procedure $\mathbf{B}$. The residue was purified by column chromatography (EtOAc/cyclohexane $20: 80)$ to give 38 (198 $\mathrm{mg}, 65 \%$ yield) as a white powder. m.p.: 111 $112{ }^{\circ} \mathrm{C} ;{ }^{1} \mathrm{H}$ NMR $\left(400 \mathrm{MHz}, d_{6}\right.$-DMSO) $\delta 0.85(3 \mathrm{H}, \mathrm{t}, J=6.8), 1.15-1.33$ $(14 \mathrm{H}, \mathrm{m}), 1.33-1.48(2 \mathrm{H}, \mathrm{m}), 3.04(2 \mathrm{H}, \mathrm{dt}$ app q, $J=6.5), 3.68(3 \mathrm{H}, \mathrm{s})$, $5.97(1 \mathrm{H}, \mathrm{t}, J=5.6, \mathrm{NH}), 6.79\left(2 \mathrm{H}^{\mathrm{ar}}, \mathrm{d}, J=9.0\right), 7.26\left(2 \mathrm{H}^{\mathrm{ar}}, \mathrm{d}, J=9.0\right), 8.15$ $(1 \mathrm{H}, \mathrm{s}, \mathrm{NH}) ;{ }^{13} \mathrm{C}$ NMR $\left(100 \mathrm{MHz}, d_{6}\right.$-DMSO) $\delta 14.0\left(\mathrm{CH}_{3}\right), 22.1\left(\mathrm{CH}_{2}\right)$, $26.4\left(\mathrm{CH}_{2}\right), 28.7\left(\mathrm{CH}_{2}\right), 28.8\left(\mathrm{CH}_{2}\right), 29.0\left(\mathrm{CH}_{2}\right), 29.1\left(\mathrm{CH}_{2}\right), 29.8\left(\mathrm{CH}_{2}\right)$, $31.3\left(\mathrm{CH}_{2}\right), 39.0\left(\mathrm{CH}_{2}\right), 55.1\left(\mathrm{CH}_{3}\right), 113.8(2 \mathrm{CH}), 119.3(2 \mathrm{CH}), 133.8$ $(\mathrm{Cq}), 153.8(\mathrm{Cq}), 155.4(\mathrm{Cq})$; IR (ATR) v max: $3325(\mathrm{~N}-\mathrm{H}), 2954,2923$, 2849, $1630(\mathrm{C}=\mathrm{O}), 1609,1560,1507,1478,1466,1244,1184,1030,829$; MS $\left(\mathrm{ESI}^{+}\right) \mathrm{m} / z 307\left([\mathrm{M}+\mathrm{H}]^{+}, 100\right), 329\left([\mathrm{M}+\mathrm{Na}]^{+}, 71\right) ; \mathrm{HRMS}_{\left(\mathrm{ESI}^{+}\right)[\mathrm{M}}$ $+\mathrm{H}]^{+}$for $\mathrm{C}_{18} \mathrm{H}_{31} \mathrm{~N}_{2} \mathrm{O}_{2}$ requires 307.2380 , found 307.2366.

\section{Procedure C: General procedure for the one-pot, solvent-free} preparation of unsymmetrical $N, N$ '-disubstituted ureas

The primary amine $(10 \mathrm{mmol})$ and trimethylsilylisocyanate $(12 \mathrm{mmol})$ were introduced in a $50 \mathrm{~mL}$-stainless steel autoclave. The reactor was tightly closed, purged three times with Argon, and then placed in a graphite bath on a magnetic stirrer and the reaction mixture was heated at $90{ }^{\circ} \mathrm{C}$ and stirred for 2 hours. After cooling to room temperature, decanal $(30 \mathrm{mmol})$ and $5 \%-\mathrm{Pd} / \mathrm{C}(2.5 \mathrm{~mol}-\%)$ were added to the reaction mixture. The reactor was tightly closed, purged three times and hydrogen pressure was introduced ( 5 bar). Then, it was placed on a magnetic stirrer and the reaction mixture was heated at $100{ }^{\circ} \mathrm{C}$ and stirred for 15 hours. After cooling to room temperature, hydrogen pressure was released, the crude mixture was dissolved in methanol, and palladium $(\mathrm{Pd} / \mathrm{C})$ was filtered under vacuum over a millipore filter and thoroughly washed with methanol several times. The filtrate was concentrated under reduced pressure and the corresponding alkylated ureas were purified by column chromatography using (EtOAc / cyclohexane). The spectroscopic data of $N, N^{\prime}$-disubstituted ureas prepared according to this procedure were in accordance with those reported before for the reductive alkylation of monosubstituted ureas; see above data for the full characterization.

Supporting Information (see footnote on the first page of this article): ${ }^{1} \mathrm{H}$ and ${ }^{13} \mathrm{C}$ NMR spectra of monosubstituted and disubstituted ureas.

\section{Acknowledgments}

The authors are grateful for the access to the GC-MS analysis at the Centre Commun de Spectroscopie de Masse (F. Albrieux, C. Duchamp and N. Henriques) and NMR facilities at Université Claude Bernard Lyon 1.

[1] a) T. P. Vishnyakova, I. A. Golubeva, E. V. Glebova, Russ. Chem. Rev. (Engl. Transl.), 1985, 54, 249-261. b) I. Gallou, Org. Prep. Proced. Int. 2007, 39, 355-383.

[2] L. dos Santos, L. A. Lima, V. Cechinel-Filho, R. Corrêa, F. de Campos Buzzi, R. J. Nunes, Bioorg. Med. Chem. 2008, 16, 85268534 .

[3] K. M. Khan, S. Saeed, M. Ali, M. Gohar, J. Zahid, A. Khan, S. Perveen, M. I. Choudhary, Bioorg. Med. Chem. 2009, 17, 2447-2451.

[4] a) S. Denoyelle, T. Chen, L. Chen, Y. Wang, E. Klosi, J. A. Halperin, B. H. Aktas, M. Chorev, Bioorg. Med. Chem. Lett. 2012, 22, 402409. b) Q.-S. Li, P.-C. Lv, H.-Q. Li, X. Lu, Z.-L. Li, B.-F. Ruan, H.L. Zhu, J. Enzyme Inhib. Med. Chem. 2012, 27, 708-714.

[5] Z. Zhang, P. R. Schreiner, Chem. Soc. Rev. 2009, 38, 1187-1198 and references cited therein

[6] N. Volz, J. Clayden, Angew. Chem. Int. Ed. 2011, 50, 12148-12155.

[7] F. Bigi, R. Maggi, G. Sartori, Green Chem. 2000, 2, 140-148.

[8] For an elegant Ritter-type reaction, see: a) H. Basavaprabhu, V. V Sureshbabu, Org. Biomol. Chem. 2012, 10, 2528-2533. b) V Panduranga, H. Basavaprabhu, V. V. Sureshbabu, Tetrahedron Lett. 2013, 54, 975-979.

[9] For an oxidative N-H/C-H cross-coupling strategy, see: X. Li, B. Li, J. You, J. Lan, Org. Biomol. Chem. 2013, 11, 1925-1928.

[10] P. Majer, R. S. Randad, J. Org. Chem. 1994, 59, 1937-1938.

[11] For recent applications, see: a) K. J. Padiya, S. Gavade, B. Kardile, M. Tiwari, S. Bajare, M. Mane, V. Gaware, S. Varghese, D. Harel, S. Kurhade, Org. Lett. 2012, 14, 2814-2817. b) P. A. Duspara, M. S Islam, A. J. Lough, R. A. Batey, J. Org. Chem. 2012, 77, 1036210368.

[12] A. R. Katritzky, D. P. M. Pleynet, B. Yang, J. Org. Chem. 1997, 62, $4155-4158$

[13] a) J. Kitteringham, M. R. Shipton, M. Voyle, Synth. Commun. 2000, 30, 1937-1943. b) A. V. Bogolubsky, S. V. Ryabukhin, S. E. Pipko, O. Lukin, A. Shivanyuk, D. Mykytenko, A. Tolmachev, Tetrahedron 2011, 67, 3619-3623.

[14] For selected examples, see: a) Y. Matsumura, Y. Satoh, O. Onomura T. Maki, J. Org. Chem. 2000, 65, 1549-1551. b) I. Gallou, M Eriksson, X. Zeng, C. Senanayake, V. Farina, J. Org. Chem. 2005, $70,6960-6963$

[15] a) I. Vauthey, F. Valot, C. Gozzi, F. Fache, M. Lemaire, Tetrahedron Lett. 2000, 41, 6347-6350. b) N. Nagaraju, G. Kuriakose, Green Chem. 2002, 4, 269-271. c) S. R. Jagtap, Y. P. Patil, A. G. Panda, B. 
M. Bhanage, Synth. Commun. 2009, 39, 2093-2100. d) F. Saliu, B. Rindone, Tetrahedron Lett. 2010, 51, 6301-6304.

[16] a) K. Kondo, S. Yokoyama, N. Miyoshi, S. Murai, N. Sonoda, Angew. Chem. Int. Ed. 1979, 18, 692-692. b) A. R. Katritzky, N. Kirichenko, B. V. Rogovoy, Arkivoc 2003, 8, 8-14.

[17] For a review regarding the carbonylation of amines with $\mathrm{CO}$, see: D. J. Díaz, A. K. Darko, L. McElwee-White, Eur. J. Org. Chem. 2007, $27,4453-4465$.

[18] a) J. C. Anderson, R. Bou Moreno, Org. Biomol. Chem. 2012, 10 , 1334-1338. b) A.-H. Liu, Y.-N. Li, L.-N. He, Pure Appl. Chem 2012, 84, 581-602. c) Z.-Z. Yang, L.-N. He, J. Gao, A.-H. Liu, B. Yu, Energy Environ. Sci. 2012, 5, 6602-6639. d) C. Wu, H. Cheng, R. Liu, Q. Wang, Y. Hao, Y. Yu, F. Zhao, Green Chem. 2010, 12 , 1811-1816. e) T. Jiang, X. Ma, Y. Zhou, S. Liang, J. Zhang, B. Han, Green Chem. 2008, 10, 465-469. f) B. M. Bhanage, S.-I. Fujita, Y. Ikushima, M. Arai, Green Chem. 2003, 5, 340-342. g) F. Shi, Y. Deng, T. SiMa, J. Peng, Y. Gu, B. Qiao, Angew. Chem; Int. Ed. 2003 42, 3257-3260. h) W. McGhee, D. Riley, K. Christ, Y. Pan, B. Parnas, J. Org. Chem. 1995, 60, 2820-2830.

[19] a) G. A. Artamkina, A. G. Sergeev, I. P. Beletskaya, Tetrahedron Lett. 2001, 42, 4381-4384. b) A. G. Sergeev, G. A. Artamkina, I. P. Beletskaya, Tetrahedron Lett. 2003, 44, 4719-4723. c) A. Abad, C. Agulló, A. C. Cuñat, C. Vilanova, Synthesis 2005, 6, 915-924. d) M. McLaughlin, M. Palucki, I. W. Davies, Org. Lett. 2006, 8, 33113314. e) M. C. Willis, R. H. Snell, A. J. Fletcher, R. L. Woodward Org. Lett. 2006, 8, 5089-5091. f) J. Clayden, U. Hennecke, Org. Lett 2008, 10, 3567-3570. g) B. J. Kotecki, D. P. Fernando, A. R. Haight, K. A. Lukin, Org. Lett. 2009, 11, 947-950. h) S. Yu, A. Haight, B. Kotecki, L. Wang, K. Lukin, D. R. Hill, J. Org. Chem. 2009, 74 9539-9542. i) S. Breitler, N. J. Oldenhuis, B. P. Fors, S. L. Buchwald, Org. Lett. 2011, 13, 3262-3265.

[20] K. A. Hackl, H. Falk, Monatsh. Chem. 1992, 123, 599-606.

[21] a) J. D. Armstrong, III, C. N. Wolfe, J. L. Keller, J. Lynch, M. Bhupathy, R. P. Volante, R. J. DeVita, Tetrahedron Lett. 1997, 38, 1531-1532. b) D. Xu, L. Ciszewski, T. Li, O. Repič, T. J. Blacklock, Tetrahedron Lett. 1998, 39, 1107-1110. c) D. Dubé, A. A. Scholte, Tetrahedron Lett. 1999, 40, 2295-2298.

[22] The reductive alkylation of thioureas has also been reported, see: L. Ciszewski, D. Xu, O. Repič, T. J. Blacklock, Tetrahedron Lett. 2004, 45, 8091-8093.

[23] F. Fache, L. Jacquot, M. Lemaire, Tetrahedron Lett. 1994, 35, 3313 3314.

[24] V. Bethmont, F. Fache, M. Lemaire, Tetrahedron Lett. 1995, 36, 4235-4236.

[25] M. Lemaire, W. Dayoub, M. Sutter, Y. Raoul, Fr. Demande, 2012, FR2969146 A120120622, PCT Int. Appl., 2012, WO2012080682 A120120621.

[26] a) Y. Shi, W. Dayoub, A. Favre-Réguillon, G.-R. Chen, M. Lemaire, Tetrahedron Lett. 2009, 50, 6891-6893. b) Y. Shi, W. Dayoub, G.-R. Chen, M. Lemaire, Green Chem. 2010, 12, 2189-2195. c) Y. Shi, W. Dayoub, G.-R. Chen, M. Lemaire, Sci. China Chem. 2010, 53, 19531956. d) M. Sutter, W. Dayoub, E. Métay, Y. Raoul, M. Lemaire, Green Chem. 2013, 15, 786-797.

[27] For reviews, see: a) S. Gomez, J. A. Peters, T. Maschmeyer, $A d v$. Synth. Catal. 2002, 344, 1037-1057. b) V. I. Tararov, A. Börner, Synlett 2005, 209-211. c) T. C. Nugent, M. El-Shazly, Adv. Synth. Catal. 2010 352, 753-819. d) J. L. Klinkenberg, J. F. Hartwig, Angew. Chem. Int. Ed. 2011, 50, 86-95. For recent examples, see: e) S. Werkmeister, K. Junge, M. Beller, Green Chem. 2012, 14, 23712374. f) A. Pagnoux-Ozherelyeva, N. Pannetier, M. D. Mbaye, S. Gaillard, J.-L. Renaud, Angew. Chem. Int. Ed. 2012, 51, 4976-4980.

[28] M. Roman, B. Andrioletti, M. Lemaire, J.-M. Bernard, J. Schwartz, P. Barbeau, Tetrahedron 2011, 67, 1506-1510.

[29] Balloon of hydrogen was not permitted for safety reasons. Very low accuracy of the manometer's autoclave for a pressure inferior to 5 bar.

Received: ((will be filled in by the editorial staff))

Published online: ((will be filled in by the editorial staff)) 


\section{Entry for the Table of Contents}

Unsymmetrical $N, N$ '-disubstituted ureas were prepared with $60-93 \%$ isolated yield from the palladiumcatalyzed reductive alkylation of monosubstituted ureas using aldehydes as alkylating agents and molecular hydrogen as a clean reductant. A onepot, solvent-free sequence was also developed from the corresponding amines.

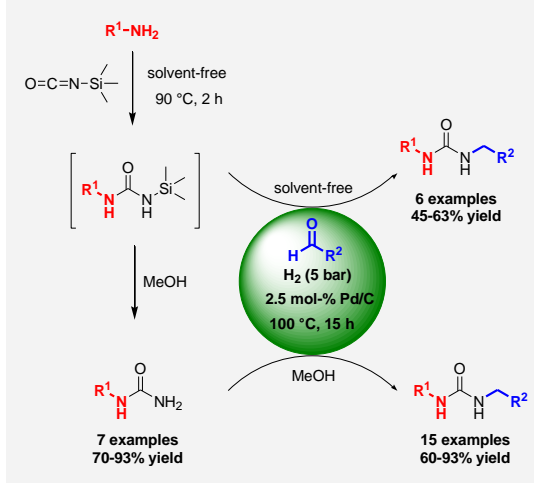

Tharwat Mohy El Dine, Simon Chapron, Marie-Christine Duclos, Nicolas Duguet, Florence Popowycz and Marc Lemaire*

One-pot, solvent-free access to unsymmetrical ureas via palladiumcatalyzed reductive alkylation using molecular hydrogen

Keywords: Unsymmetrical Disubstituted Ureas / Reductive Alkylation / Palladium / Hydrogen 
